\title{
Modifier variant of METTL13 suppresses human CAB1-associated profound deafness
}

\author{
Rizwan Yousaf, ${ }^{1}$ Zubair M. Ahmed, ${ }^{1}$ Arnaud P.J. Giese, ${ }^{1}$ Robert J. Morell, ${ }^{2}$ Ayala Lagziel, ${ }^{2}$ Alain Dabdoub, ${ }^{3}$ Edward R. Wilcox, ${ }^{2}$ \\ Sheikh Riazuddin,, ${ }^{4,5}$ Thomas B. Friedman, ${ }^{2}$ and Saima Riazuddin ${ }^{1,4,5}$

\begin{abstract}
'Laboratory of Molecular Cenetics, Department of Otorhinolaryngology - Head and Neck Surgery, University of Maryland, Baltimore, Maryland, USA. ${ }^{2}$ Laboratory of Molecular Cenetics, National Institute on Deafness and Other Communication Disorders (NIDCD), NIH, Bethesda, Maryland, USA. ${ }^{3}$ Laboratory of Cochlear Development, NIDCD, NIH, Bethesda, Maryland, USA. ${ }^{4}$ Allama Iqbal Medical College,
\end{abstract} \\ University of Health Sciences, Lahore, Pakistan. ${ }^{5}$ Shaheed Zulfiqar Ali Bhutto Medical University, Pakistan Institute of Medical Sciences, Islamabad, Pakistan.
}

\begin{abstract}
A modifier variant can abrogate the risk of a monogenic disorder. DFNM1 is a locus on chromosome 1 encoding a dominant suppressor of human DFNB26 recessive, profound deafness. Here, we report that DFNB26 is associated with a substitution (p.Gly116Clu) in the pleckstrin homology domain of GRB2-associated binding protein 1 (CAB1), an essential scaffold in the MET proto-oncogene, receptor tyrosine kinase/HGF (MET/HGF) pathway. A dominant substitution (p.Arg544GIn) of METTL13, encoding a predicted methyltransferase, is the DFNM1 suppressor of CAB1-associated deafness. In zebrafish, human METTL13 mRNA harboring the modifier allele rescued the GAB1-associated morphant phenotype. In mice, GAB1 and METTL13 colocalized in auditory sensory neurons, and METTL13 coimmunoprecipitated with GAB1 and SPRY2, indicating at least a tripartite complex. Expression of MET-signaling genes in human lymphoblastoid cells of individuals homozygous for p.Cly116Clu GAB1 revealed dysregulation of HCF, MET, SHP2, and SPRY2, all of which have reported variants associated with deafness. However, SPRY2 was not dysregulated in normal-hearing humans homozygous for both the GAB1 DFNB26 deafness variant and the dominant METTL13 deafness suppressor, indicating a plausible mechanism of suppression. Identification of METTL13-based modification of MET signaling offers a potential therapeutic strategy for a wide range of associated hearing disorders. Furthermore, MET signaling is essential for diverse functions in many tissues including the inner ear. Therefore, identification of the modifier of MET signaling is likely to have broad clinical implications.
\end{abstract}

\section{Introduction}

A modifier variant can enhance or suppress the phenotype of a monogenic disorder as well as alter the onset, severity, or progression of the phenotype (1-5). The site of action of modifiers can be at any point from transcription to posttranslational modification of the target genes. Genetically mapping a modifier variant in humans is challenging because of environmental factors and the usual limitations in the number of family subjects with, for example, a nonpenetrant or enhanced phenotype. To date, only 3 modifier loci/genes for human hereditary hearing loss have been reported (6-9). Homoplasmic alleles of human mitochondrial 12S rRNA are associated with nonsyndromic deafness. The hearing loss phenotype is modified by aminoglycoside exposure that reduces the age of hearing loss onset $(10,11)$. Additionally, a variant of tRNA 5-methylaminomethyl2-thiouridylate methyltransferase (TRMU) is implicated as a nuclear modifier of human 12S rRNA variant-associated deafness (Online Mendelian Inheritance in Man [OMIM] 580000) (6). Similarly, a hypomorphic allele of the plasma membrane calcium pump PMCA2 modulates the severity of cadherin 23linked hearing loss (9).

Conflict of interest: The authors have declared that no conflict of interest exists Submitted: September 6, 2017; Accepted: January 30, 2018. Reference information: / Clin Invest. 2018;128(4):1509-1522. https://doi.org/10.1172/JCI97350.
In 2000, we genetically mapped the hearing loss modifier locus DFNM1 (OMIM 605429) to a $5.6 \mathrm{cM}$ region on chromosome 1q24, with a logarithm of the odds (LOD) score of 4.31 . DFNM1 is an unlinked dominant suppressor of DFNB26 deafness (OMIM 605428), which mapped to a $1.5 \mathrm{cM}$ interval of chromosome $4 \mathrm{q} 31$, with a LOD score of 8.10 (7). Here, we report the identification and characterization of the variants associated with hearing loss DFNB26 and the modifier allele of METTL13 at the DFNM1 locus. Using a combination of Sanger sequencing and exome enrichment followed by massive parallel sequencing of genomic DNA and mRNA from affected and nonpenetrant individuals, we identified a DFNB26 hearing loss-linked allele in the pleckstrin homology $(\mathrm{PH})$ domain of the adapter protein of the MET proto-oncogene receptor tyrosine kinase-signaling pathway (MET-signaling pathway), GRB2-associated binding protein 1 (GAB1). At the DFNM1 locus, we also identified a dominant allele of METTL13, encoding a predicted methyltransferase. Our in vitro and in vivo studies reveal that GAB1 and METTL13, which function in the MET/HGF-signaling pathway, are necessary for normal functioning of the auditory system.

\section{Results}

Identification of DFNB26 and DFNM1 genes. Under the assumption that variants at the DFNB26 and DFNM1 loci explain the auditory phenotype and the suppressor of deafness segregating in family PK-2, respectively (Figure 1A), we next sought to identify 
A

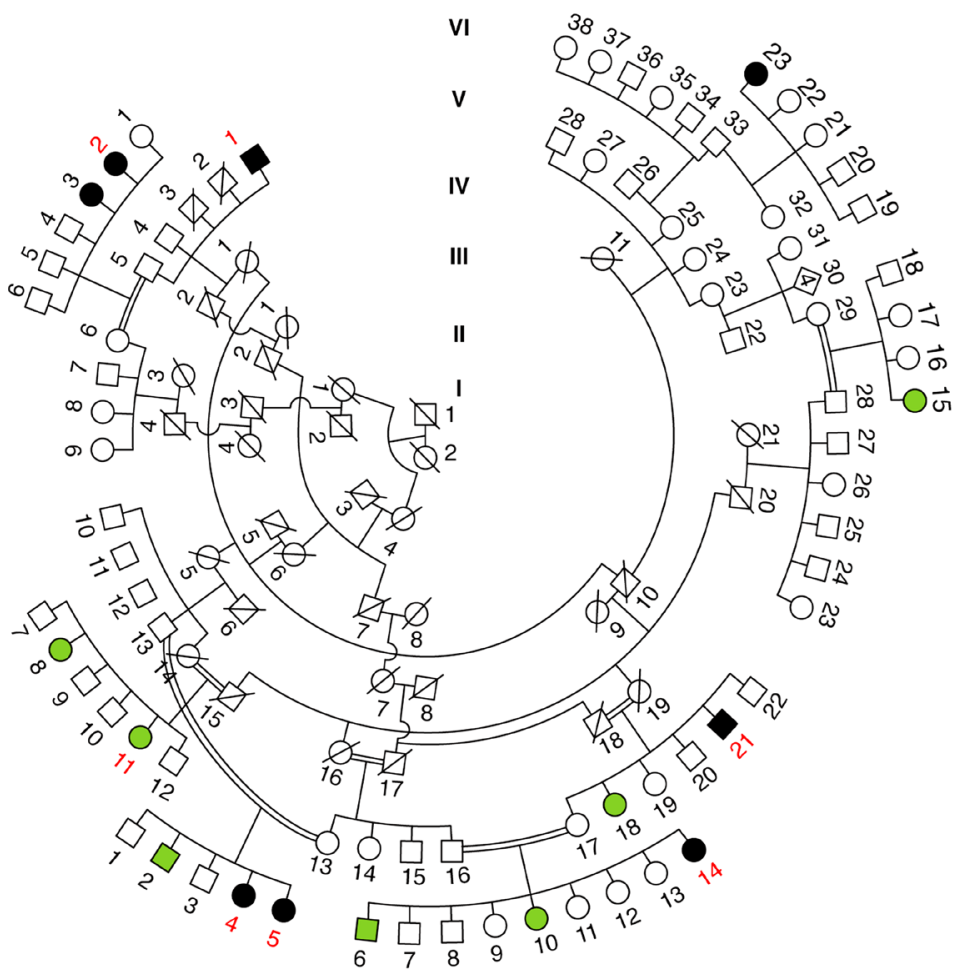

B

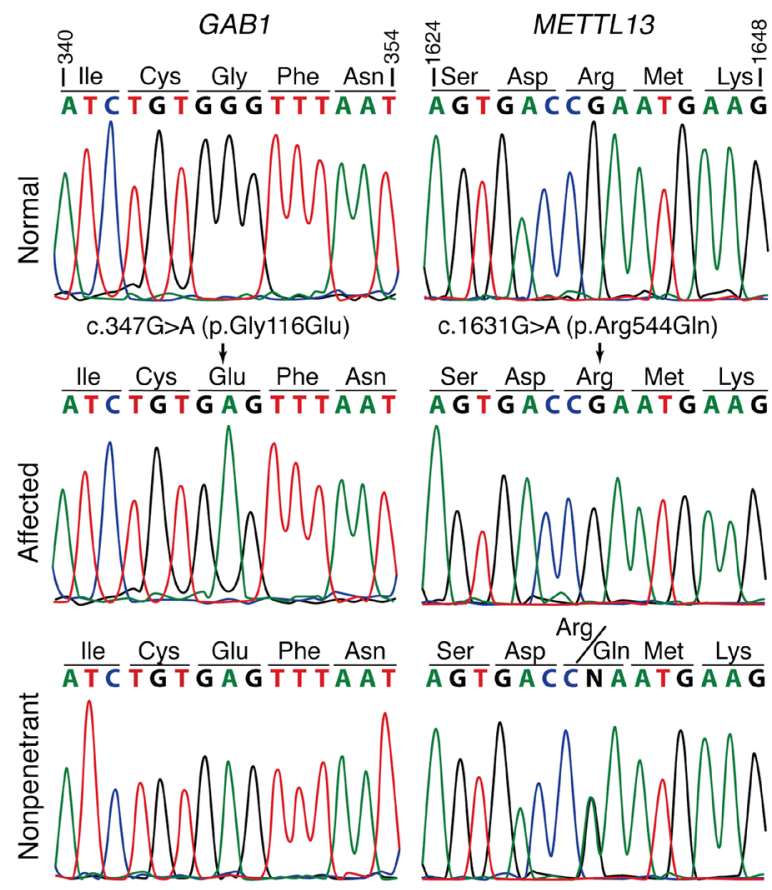

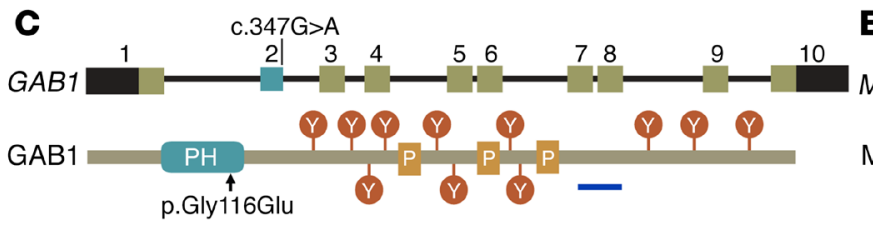

D GAB1

E

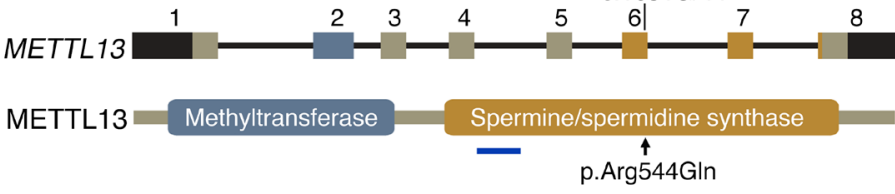

F METTL13

p.Arg544

H. sapiens VATQWFGFSQSDRMKVHIADGLDYIA

P. troglodytes VATQWFGF SQSDRMKVHIADGLDYIA

M. mulatta VATQWFGFSQSDRMKVHIADGLDYIA

M. murinus VATQWFGFSQSDRMKVHIADGLDYIT

M. musculus VATQWFGFSQSDRMKVHIADGLDYIT

R. norvegicus VATQWFGFAQSDRMKVHIADGLDYVT

C. porcellus VATQWFGFSQSDRMKVHIADGLDYIT

D. rerio VAQTWFGFQIDDRLKVTLGDGLEHIT

D. melanogaster VAEQYFELKQDKRFHVVIDDGLDFVE

Figure 1. Variants of CAB1 and METTL13 associated with DFNB26 deafness and the unlinked DFNM1 dominant suppressor of DFNB26 deafness, respectively. (A) PK-2 family segregating DFNB26-linked hearing loss and the DFNM1 modifier gene. Black symbols indicate affected individuals homozygous for the GAB1 variant. Green symbols indicate normal-hearing (nonpenetrant) individuals homozygous for the GAB1 variant and the METTL13 dominant modifier allele. The 7 genomic DNA samples used for WES are indicated by red numbers. (B) Nucleotide sequence chromatograms of exon 2 of $C A B 1$ showing the WT sequence of a normal-hearing individual, while the affected and nonpenetrant individuals are homozygous for c.347G>A allele. Also shown are the nucleotide sequence chromatograms of exon 6 of METTL13 from a normal-hearing individual, an affected individual who is homozygous for the WT allele, and a nonpenetrant individual who is heterozygous for c.1647C>A allele of METTL13. (C) The longest transcript of human GAB1 has 10 exons. The c.347G > A variant is located in constitutive exon 2. The GAB1 protein has a PH domain, which harbors the p.Gly116Glu substitution. The position of tyrosine phosphorylation sites $(Y)$ and 2 proline-rich regions $(P)$ are shown along with the peptides (blue bar) used for antibody generation for each protein. (D) The glycine residue at amino acid position 116 of human GAB1 is conserved. (E) The longest transcript of human METTL13 has 8 exons. The c.1631G>A modifier variant is in exon 6 . METTL13 protein is predicted to have methyltransferase and spermine/spermidine synthase domains. The antigen used for producing polyclonal antibodies is indicated by a blue bar below the METTL13 structure. (F) METTL13 arginine 544 is conserved in a wide variety of species.

the 2 genes by Sanger sequencing all annotated genes within the DFNB26 and DFNM1 linkage intervals using genomic DNA from affected and nonpenetrant individuals. A homozygous transition variant c.347G $>$ A in exon 2 of GAB1 (NM 207123) was identified in deaf and nonpenetrant individuals. The c.347G>A transition variant is predicted to result in a substitution of glutamate for a conserved glycine (p.Gly116Glu) in the PH domain of GAB1 (Figure 1, B-D, and Supplemental Figure 1; supplemental mate- 
Table 1. Bioinformatics evaluation of variants found in CAB1 and METTL13 genes

$\begin{array}{lcc} & \text { GAB1 } & \text { METTL13 } \\ \text { hg19 coordinates } & \text { Chr4:144336904 } & \text { Chr1:171761313 } \\ \text { GenBank accession no. } & \text { NM_207123 } & \text { NM_015935 } \\ \text { Nucleotide variant } & \text { c.347G>A } & \text { c.1631G>A } \\ \text { Amino acid substitution } & \text { p.Gly116Glu } & \text { p.Arg544GIn } \\ \text { PhyloPA } & 9.975 & 9.424 \\ \text { GERPB } & 5.94 & 5.80 \\ \text { PolyPhen-2 } & \text { Disease-causing } & \text { Disease-causing } \\ \text { PROVEAN } & \text { Deleterious } & \text { Deleterious } \\ \text { SIFT } & \text { Damaging } & \text { Damaging } \\ \text { CADD } & \text { 28 (Deleterious) } & \text { 35 (Deleterious) } \\ \text { MutationTaster } & \text { Disease-causing } & \text { Disease-causing } \\ \text { Mutation Assessor } & \text { Medium } & \text { Medium } \\ \text { SNPs3D } & -1.31 \text { (Pathogenic) } & -2.22 \text { (Pathogenic) } \\ \text { LRT } & \text { Deleterious } & \text { Deleterious }\end{array}$

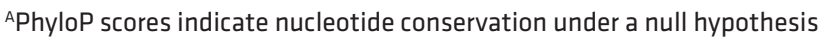
of neutral evolution, in which conserved sites are assigned positive scores. ${ }^{B}$ Genomic Evolutionary Rate Profiling (GERP) provides position-specific estimates of evolutionary constraint (scores: -12.36 to 6.18). Positive scores are indicative of fewer substitutions than the average neutral site. CADD, combined annotation-dependent depletion; LRT, likelihood ratio test; SIFT, sorting intolerant from tolerant. PolyPhen-2 (http://genetics.bwh.harvard. edu/pph2/); Protein Variation Effect Analyzer (PROVEAN; http://provean. jcvi.org/index.php); MutationTaster (http://www.mutationtaster.org/); Mutation Assessor (http://mutationassessor.org/v1/).

rial available online with this article; https://doi.org/10.1172/ JCI97350DS1). No carriers of the c.347G>A variant were found among 380 control chromosomes from Pakistani individuals or in 192 normal-hearing individuals from India. The c.347G >A variant of $G A B 1$ is not listed in the 1000 Genomes database (12), nor is it identified in 6,500 individuals in the National Heart, Lung, and Blood Institute Exome Sequencing Project (NHLBI-ESP) exome variant server (13) or in 60,706 individuals in the Exome Aggregation Consortium (ExAC) data set (14) (Supplemental Table 1). We used 8 bioinformatics tools to evaluate functionality in silico, and all of them predicted that p.Gly116Glu is deleterious for $G A B 1$ function (Table 1).

The exons and their flanking sequence of the 33 annotated genes at the DFNM1 modifier locus were Sanger sequenced, revealing a heterozygous c.1631G $>\mathrm{A}$ transition change in $M E T$ TL13 (NM_015935) (Figure 1B). The c.1631G $>$ A allele cosegregated with the DFNM1-linked haplotype in the PK-2 family, and all deaf individuals were homozygous for the WT allele of METTL13. The c.1631G $>$ A variant is predicted to substitute a glutamine for a highly conserved arginine (p.Arg544Gln) in the spermine/spermidine synthase domain of METTL13 (Figure 1, E and F). No carriers of the c. $1631 \mathrm{G}>\mathrm{A}$ change were found among 384 chromosomes from Pakistani controls, 192 from Indian controls, or 172 chromosomes from the Human Diversity Panel and in the 1000 Genome database. One carrier of African American ancestry was found among 13,006 chromosomes listed in the NHLBI-ESP6500 variant database, and three carriers were among the 60,706 European individuals listed in the ExAC database (Supplemental Table 1).

Our Sanger sequencing studies of all exons and the adjacent flanking sequence of the annotated genes within the linkage interval of DFNB26 and DFNM1 loci only revealed the above-described candidate variants segregating with the phenotype in family PK-2. However, we considered an alternative hypothesis that intrafamilial genetic heterogeneity might account for the hearing loss in family PK-2 (15). To test this hypothesis that the hearing loss segregating in different sibships of family PK-2 might be due to pathogenic variants in different genes, we performed whole-exome sequencing (WES) on 6 deaf individuals from 6 sibships of family PK-2, 1 nonpenetrant, normal-hearing individual (Figure 1A), and 1 unrelated normal control individual, using the NimbleGen SeqCap EZ Exome Library v2.0 (Roche). These WES libraries were sequenced using an Illumina HiSeq2000. All variants found in the WES data are summarized in Table 2. For each sample subjected to WES, we found many genes with homozygous or compound heterozygous, nonsynonymous changes (Table 2 and Supplemental Tables 2 and 3). We performed bioinformatics analyses, which identified 5 genes with potential pathogenic variants in each affected individual, including the previously discovered c.347G >A variant in GAB1 (Table 2). Segregation analysis of each of these variants in their respective sibships revealed that only the c. $347 \mathrm{G}>\mathrm{A}$ change in $G A B 1$ cosegregated with deafness within each sibship. WES data did not reveal any potential pathogenic variants shared among affected individuals, except for the c.347G $>$ A allele in GAB1 at the DFNB26 locus (Table 2 and Supplemental Tables 2 and 3). Thus, the WES results support the supposition that hearing loss in family $\mathrm{PK}-2$ is due to a recessive missense pathogenic variant in GAB1 at the DFNB26 locus. For the DFNM1 linkage interval, the only coding variant that cosegregated with the DFNM1-linked haplotype in family PK-2 was c.1631G>A in METTL13 (Table 2).

To further evaluate the biological effects of these variants at the transcriptional level, we established lymphoblastoid cell lines from 3 affected individuals, 2 nonpenetrant individuals, and 1 normalhearing individual of family PK-2. We extracted the RNA from these cell lines and generated cDNA libraries. Sanger sequencing of GAB1 and METTL13 cDNAs in these libraries confirmed the presence of c. $347 \mathrm{G}>\mathrm{A}$ and c. $1631 \mathrm{G}>\mathrm{A}$ variants.

The DFNM1 modifier allele attenuates the effect of mutant GAB1 in zebrafish. The zebrafish genome has single homolog of human GAB1 (62\% amino acid sequence identity, 75\% similarity) and METTL13 (54\% identity, 70\% similarity). In zebrafish whole embryos, we detected the expression of gab1 and mettl13 by real-time PCR throughout development (Figure 2, A and B), including in the developing brain and inner ear (Figure 2, C-J). A translation-blocking morpholino (MO) targeting zebrafish gab1 was injected into 1- to 2-cell-stage embryos. Masked scoring of live embryos at the 10- to 12-somite stage revealed mild-to-severe developmental defects (Figure 2, K and L). To rule out MO toxicity, we used various dilutions and also blocked the p53 pathway via coinjection of a tP53-targeting MO (16). Furthermore, a second MO directed against the intron 2/exon 3 splice junction of gab1 revealed the same developmental deficits (Supplemental Figure 2A). Thus, morphant developmental deficits appear to be specific to the knockdown of gab1 expression. 
Table 2. Summary of variants identified by WES in 6 deaf individuals (affected) and 1 nonpenetrant individual of family PK-2

Exome-sequencing data

\begin{tabular}{|c|c|c|c|c|c|c|c|c|}
\hline & Affected VII: 5 & Affected VII: $\mathbf{4}$ & Affected VII: 14 & Affected VI: 2 & Affected VI: 21 & Affected V: 1 & Nonpenetrant & Control $^{\mathrm{B}}$ \\
\hline $\begin{array}{l}\text { Total changes }{ }^{\mathrm{A}} \text { (changes at DFNB26, } \\
\text { DFNM1 loci) }\end{array}$ & $\begin{array}{l}58,193 \\
(50,97)\end{array}$ & $\begin{array}{l}49,032 \\
(25,117)\end{array}$ & $\begin{array}{l}48,060 \\
(33,92)\end{array}$ & $\begin{array}{l}49,746 \\
(33,131)\end{array}$ & $\begin{array}{c}49,783 \\
(36,126)\end{array}$ & $\begin{array}{c}49,288 \\
(26,100)\end{array}$ & $\begin{array}{l}62,654 \\
(56,148)\end{array}$ & $\begin{array}{l}60,200 \\
(51,166)\end{array}$ \\
\hline Not in dbSNP135 with MAF $\geq 0.1$ & $2,493(2,8)$ & $1,237(1,4)$ & $1,253(1,7)$ & $1,256(1,1)$ & $1,252(1,0)$ & $1,294(1,4)$ & $2,708(4,11)$ & $2,665(1,4)$ \\
\hline Nonsyn/SS/ins/del & $596(1,0)$ & $287(1,0)$ & $325(1,1)$ & $291(1,0)$ & $314(1,0)$ & $328(1,0)$ & $592(1,1)$ & $675(0,1)$ \\
\hline Not present in the control & $521(1,0)$ & $277(1,0)$ & $319(1,1)$ & $286(1,0)$ & $307(1,0)$ & $316(1,0)$ & $506(1,1)$ & - \\
\hline $\begin{array}{l}\text { Cenes with homozygous/compound } \\
\text { heterozygous changes }\end{array}$ & $31(1,0)$ & $14(1,0)$ & $45(1,1)$ & $14(1,0)$ & $21(1,0)$ & $21(1,0)$ & $65(1,1)$ & $20(0,0)$ \\
\hline $\begin{array}{l}\text { Not present in } 1000 \text { Cenomes/NHLBI } \\
\text { exome }\end{array}$ & $22(1,0)$ & $11(1,0)$ & $36(1,1)$ & $21(1,0)$ & $22(1,0)$ & $17(1,0)$ & $12(1,1)$ & $12(0,0)$ \\
\hline $\begin{array}{l}\text { Potential pathological changes in } \\
\text { known deafness genes }\end{array}$ & 0 & 0 & $1^{D}$ & 0 & 0 & 0 & 0 & 0 \\
\hline $\begin{array}{l}\text { Cenes with predicted pathogenic } \\
\text { changes }^{\mathrm{E}}\end{array}$ & $6(1,0)$ & $3(1,0)$ & $8(1,0)$ & $2(1,0)$ & $3(1,0)$ & $8(1,0)$ & $5(1,1)$ & $0(0,0)$ \\
\hline $\begin{array}{l}\text { Present in all the hearing-impaired } \\
\text { individuals }^{F}\end{array}$ & $1(1,0)$ & $1(1,0)$ & $1(1,0)$ & $1(1,0)$ & $1(1,0)$ & $1(1,0)$ & $1(1,1)$ & - \\
\hline
\end{tabular}

${ }^{A}$ Changes shown with a minimum of $10 \times$ coverage. ${ }^{B}$ Normal-hearing, ethnically matched sample. 'Heterozygous changes were observed in the sample from the nonpenetrant individual. ${ }^{\mathrm{D} A D C Y 1}$ : p.(Ala902Thr). EPredicted to be deleterious by at least 2 of the 3 prediction programs: PolyPhen-2 (62), SNPs3D (63), and MutationTaster (64). FSegregation analysis among the affected individuals through Sanger sequencing. Nonsyn, nonsynonymous; SS, splice site; ins, insertion; del, deletion. MAF, minor allele frequency.

The gab1 morphant phenotypes in zebrafish were subdivided into 3 classes: mildly altered (class I), moderately altered (class II), and severely altered (class III) (Figure 2K). Morphants with the mild phenotype (class I) only showed a defect in eye formation, ranging from malformed to completely absent. Morphants with the moderate phenotype (class II) had delayed development, with embryos stalled at the budding stage. Severe phenotype morphants (class III) had greatly delayed development, with embryos arrested at the $50 \%$ epiboly to late epiboly stages. Importantly, we were able to rescue these morphant phenotypes with a coinjection of human WT GAB1 mRNA (1 ng; GenBank accession no. NM_207123) (Figure 2L). A significantly (ANOVA, $P<0.005$ ) larger number of embryos were normal after coinjection of human WT GAB1 mRNA with the gab1 MO (5-bp mismatch between the MO sequence and human $G A B 1$ when compared with the total number of normal embryos found after injection of the gab1 MO alone (Figure 2L). Similarly, in the rescue experiment, significantly $(P<0.005)$ fewer embryos were in the class III category as compared with the gab1 MO alone (Figure 2L). However, rescue of the morphant phenotypes (all classes, including normal) by coinjection of the gab1 MO and human GAB1 mRNA with the c.347G>A (p.Gly116Glu) variant was not statistically significant when compared with the corresponding injection categories of the gab1 MO alone, suggesting the pathogenic nature of the p.Gly116Glu variant (Figure 2L). Compared with only gab1 MO-injected embryos, we observed an increase in normal-appearing embryos (Figure 2L), suggesting that p.Gly116Glu is a hypomorphic allele of GAB1. A complete knockout of Gab1 function in mice is embryonically lethal (17).

Next, we investigated the modifier allele and repeated the in vivo rescue assays with human GAB1-mutant mRNA (1 ng) along with human METTL13 mRNA (1 ng) harboring the c.1634G>A modifier variant. We observed no rescue of the morphant phe- notype (Supplemental Figure 3). Since the human METTL13mutant allele in humans is heterozygous in nature, we performed the rescue experiment with lower concentrations, down to $125 \mathrm{pg}$. We observed a significant rescue when 250 pg human METTL13 mRNA with the modifier variant mRNA was injected along with human GAB1-mutant mRNA, especially in the severe class III phenotype $(P<0.005)$ (Figure $2 \mathrm{~L}$ and Supplemental Figure $2 \mathrm{~B})$. We did not detect rescue when we used WT human METTL13 mRNA (NM_015935) in combination with mutant human GAB1 mRNA (Figure 2L). In agreement with the genetic observations in family PK-2, these in vivo zebrafish data support a modifying role of suppressor human METTL13 mRNA to partially rescue the phenotype associated with the human GAB1-mutant allele.

DFNB26 allele effects on the lipid-binding ability of the GAB1 PH domain. Bioinformatics tools suggested a pathogenic effect of the DFNB26 allele on the function of GAB1 (Table 1). To experimentally evaluate this finding, we investigated the effect of the DFNB26 (p.Gly116Glu) allele on the lipid-binding function of the GAB1PH domain. We purified the WT and mutant GST-PH domains (amino acid residues 24-120; NP_997006) of GAB1 and examined the ability to bind phosphatidylinositol lipids. We incubated equal amounts of GST-GAB1 WT protein and GST-GAB1 with the p.Gly116Glu variant harboring the $\mathrm{PH}$ domain (Figure 3A) with membranes containing serial dilution of various phosphoinositides (Figure 3B). Data from 3 independent experiments, each with different fresh preparations of proteins, revealed significantly lower (ANOVA, $P<0.01$ ) amounts of GAB1 PH domain harboring the p.Gly116Glu variant bound to PtdIns $(3,4,5) \mathrm{P} 3, \operatorname{PtdIns}(4,5) \mathrm{P} 2$, PtdIns(3)P, and PtdIns(4)P phoshoinositides compared with the GAB1 WT PH domain (Figure 3C).

GAB1 and METTL13 are colocalized in the mouse inner ear. Next, we asked whether METTL13 and GAB1 are expressed in the same 
A

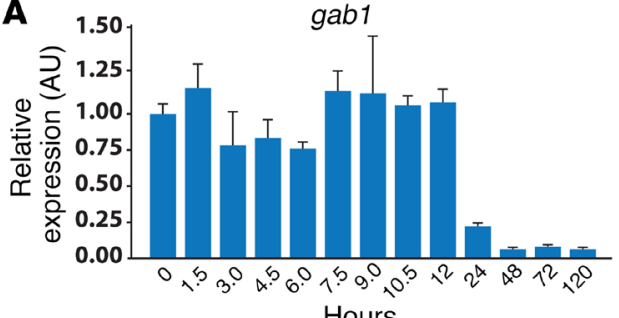

Hours
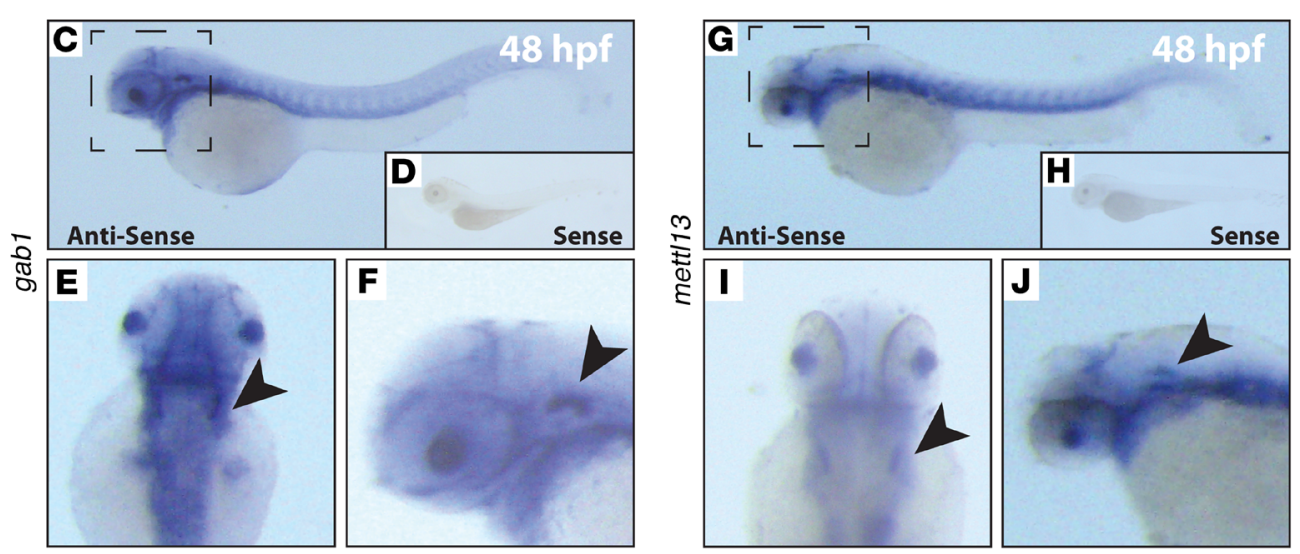

K

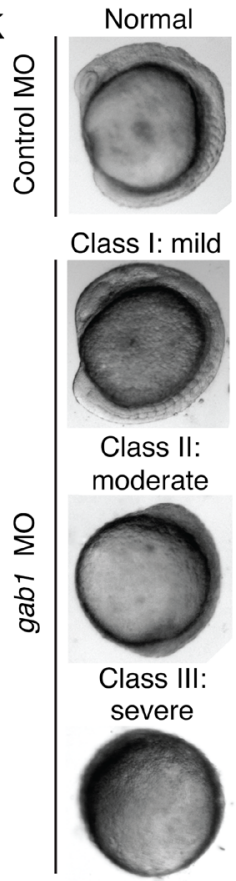

B

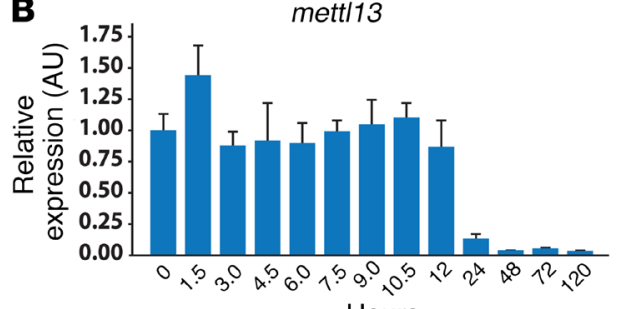

Hours

$\mathbf{L}$

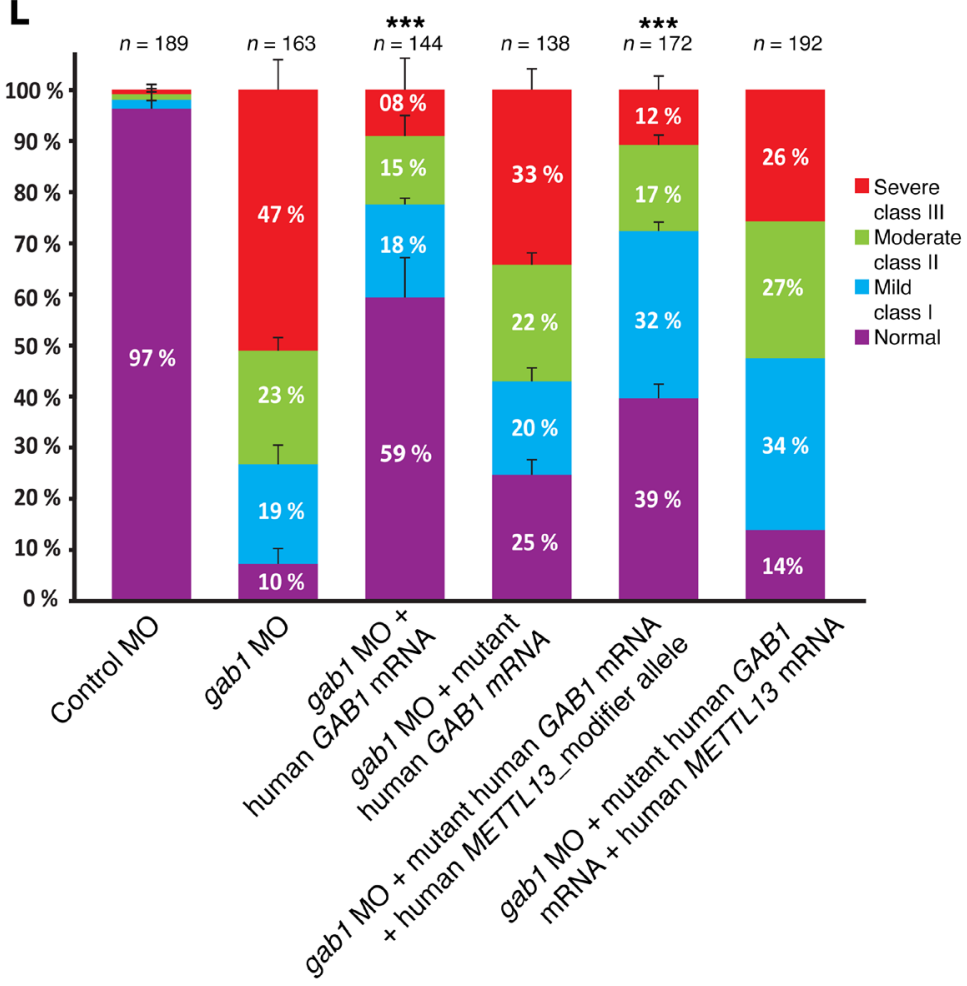

Figure 2. The human METTL13 modifier variant suppresses the effect of the $G A B 1$ variant in vivo. ( $A$ and

B) Expression in zebrafish embryos of WT gab1 and mett/13 mRNA normalized against expression of gapdh. Expression of gab1 and mett/13 mRNA was easily detectable during early development (up to 12 hours), but subsequently declined. (C-J) RNA ISH of gab1 and mett/13 in whole zebrafish embryos at 48 hours post fertilization (hpf) shows expression in many tissues, but especially in the brain region and the developing inner ear (arrowheads). (K) Lateral views of normal and gab1 morphants. MO suppression of gab1 resulted in developmental defects that were divided into 3 classes. Class I: defect in eye formation; class II: delayed development with embryos stalled at the budding stage; class III: marked developmental delay with embryos arrested at $50 \%$ epiboly to late epiboly. (L) In vivo rescue assay of gab1 $\mathrm{MO}$ with human mRNA. Coinjection of $1 \mathrm{ng}$ WT human $C A B 1$ mRNA with zebrafish gab1 mRNA translation-blocking $\mathrm{MO}$ resulted in significant rescue at the 10 - to 12-somite stage, whereas mRNA encoding the hypomorphic p.Gly116Glu variant resulted in partial rescue. Coinjection of human fulllength METTL13 mRNA encoding the p.Arg544GIn-suppressor variant of DFNB26 deafness along with $C A B 1$ mutant mRNA and gab1 MO significantly attenuated the phenotype at the 10 - to 12-somite stage, while the WT human METTL13 mRNA did not. The number of embryos phenotyped for each experiment is shown. ${ }^{* *} P$ $<0.005$, by 1 -way ANOVA for rescue versus gab1 MO normal and severe (class III) morphants. cell types in the inner ear and might interact with one another. We first examined the expression of Gab1 in the developing inner ear using normal-hearing mice heterozygous for a $\mathrm{Gab1}^{+/ \mathrm{LacZ}}$-targeted allele. Homozygotes for this Gab1-targeted allele are embryonically lethal (17). $\beta$-Gal staining of the cochlea from $\mathrm{Gab1}^{+/ \mathrm{Lacz}}$ mice on PO showed labeling of spiral ganglion, stria vascularis, spiral prominence, internal and external sulcus cells, and Reissner's membrane (Figure 4, A and D). Likewise, in the vestibular end organs (ampulla and utricle), we observed $\beta$-gal activity only in the sensory epithelium and transition cells (Figure 4, B and C).

To determine the expression of Mettl13 in the developing mouse inner ear (E11.5, E15.5, and P5), we performed ISH using a fluorescence-tagged, full-length cDNA probe (NM_144877.1). Mettl13 was expressed in the mouse otocyst at E11.5 (data not shown) and in the spiral ganglion region at E16 (Figure 4F). At P5, the epithelial cells of the cochlear duct including the stria 

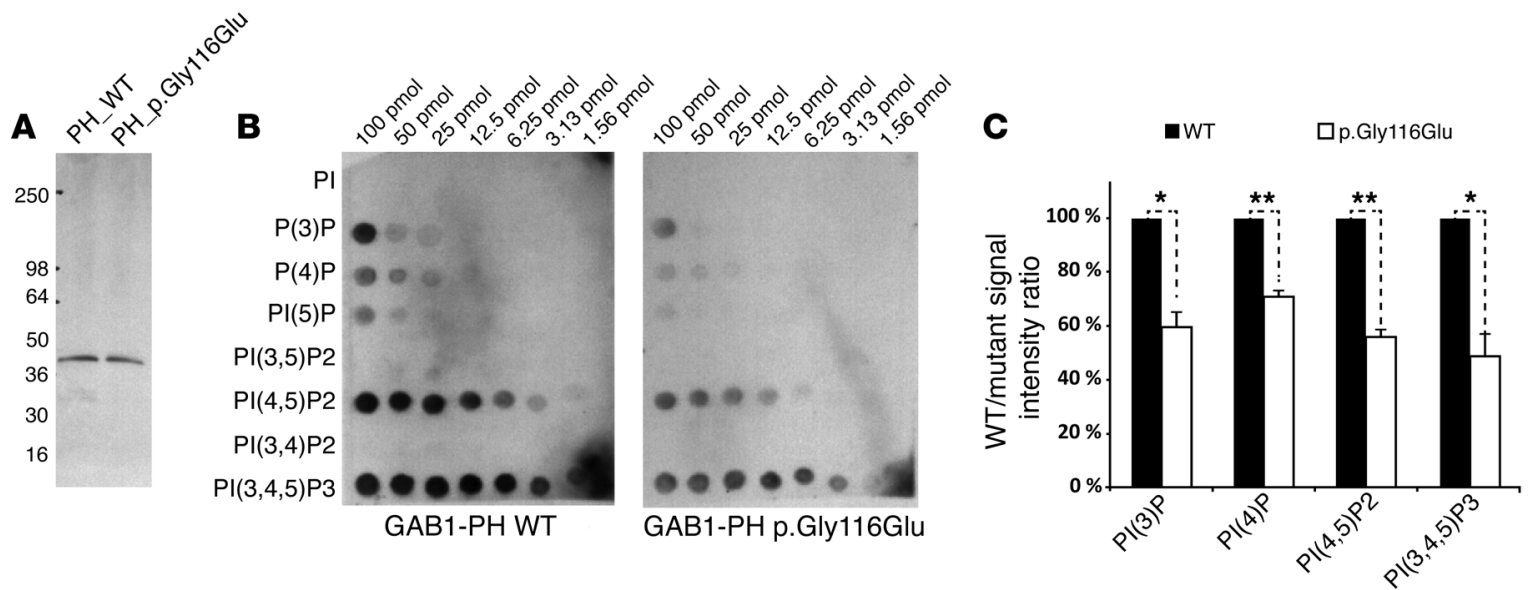

Figure 3. GAB1 harboring the p.Gly116Glu substitution has reduced binding affinity for lipids. (A) Western blot data show equal amounts of GAB1 normal and mutant PH domains that were subsequently used in the overlay assay. (B) Overlay assay with WT GAB1-PH domain protein and membrane (PIP array; Echelon Biosciences) spotted with increasing amounts of phosphorylated PI shows preferential binding to phosphatigylinosital 3,4,5-triphosphate [Ptdlns(3,4,5)P3, Ptdlns(4,5)P2 and Ptdlns(3)P], as reported previously (61). No difference was observed in the binding pattern of GAB1-PH domain harboring the p.Gly116Glu allele with Ptdlns. However, p.Gly116Glu substitution reduced the binding of the PH domain to Ptdlns(3,4,5)P3. Overlay assays with WT and mutant proteins were performed at least 3 times with 3 fresh protein preparations. (C) Graphical representation of the normalized overlay assay results after densitometric quantification of the 100 pmol spots only. The data are shown as a decrease in the relative percentage difference of binding affinity of the mutant PH domain protein when compared with the GAB1 WT PH domain. The signal intensity of each spot was first normalized against the blot background, and then all the results were further normalized against the $\mathrm{PI}(5) \mathrm{P}$ signal intensity for the WT GAB1-PH domain. On average, a $42 \%\left({ }^{* *} P<\right.$ 0.01 ) decrease in the binding of the mutant $\mathrm{PH}$ domain protein to $\mathrm{Ptd} \operatorname{lns}(3,4,5) \mathrm{P} 3$ was observed, as well as reductions of $44 \%, 40 \%$, and $30 \%\left({ }^{*} P<0.05\right)$ in binding to Ptdlns(4,5)P2, PtdIns(3)P, and PtdIns(4)P, respectively. $P$ values were determined by 2-tailed Student's $t$ test. Data represent the mean \pm SEM.

vascularis, spiral prominence, Reissner's membrane, inner and outer sulcus cells, and spiral ganglion cells showed expression of Mettl13 (Figure 4E), while the negative control (sense probe) showed no staining (Figure 4G). These data indicate that Gab1 and Mettll3 are expressed in the spiral ganglion, stria vascularis, spiral prominence, internal and external sulcus cells, and Reissner's membrane of the cochlea. Transcriptome analyses confirmed the expression of Gab1 and Mettl13 in various cell types within the mouse inner ear (Supplemental Table 4).

To determine the cellular and subcellular distribution in the adult inner ear, we used custom antisera against mouse GAB1 (PB296) and METTL13 (PB447, PB448) (Supplemental Figure 3) and performed immunofluorescence confocal microscopy on inner ear cells from WT C57BL6/J mice. We observed that both GAB1 and METTL13 colocalized in the cochlear duct, spiral limbus region, efferent and afferent nerves (Supplemental Figure 4), and in spiral ganglion neurons (Figure 5). We observed similar expression levels in vestibular neurons as well (data not shown).

METTL13 interacts in vitro with GAB1 and SPRY2. Next, we investigated the possibility that METTL13 and GAB1 proteins interact with one another. First, we used the recently reported nanoscale pulldown assay (NanoSPD) within living cells using a MYO10 ${ }^{\text {NANOTRAP }}$ construct (18). In COS-7 cells, we used MYO10 ${ }^{\text {NANOTRAP }}$ to transport GAB1-GFP to the filapodia tips (Figure $6 \mathrm{~A}$ and Supplemental Figure 5). Intriguingly, when coexpressed, both WT and mutant GAB1-GFP trafficked METTL13 proteins to the filopodia tips (Figure 6A), indicating their interaction. Insertion of the DFNB26 and DFNM1 variants into GAB1 and METTL13, respectively, did not affect their interaction (Figure 6A). Furthermore, GAB1, METTL13, and SPROUTY 2, also a member of the MET/HGF-signaling pathway, formed a tripartite complex within COS-7 cells (Figure 6, B and C). To further confirm these interactions, we performed co-IP using Flag-tagged METTL13 (METTL13-Flag), HA-tagged GAB1 (GAB1-HA), and Myc-tagged SPRY2 (SPRY2-Myc) (Figure 7, A and B, and Supplemental Figure 6). Intriguingly, WT or mutant GAB1-HA coimmunoprecipitated in the presence of either the WT or mutant METTL13 protein. Similarly, without bias for WT or mutant protein, METTL13, but not GAB1, was able to pull down SPROUTY2 protein (Figure 7, A and B). These studies suggest that METTL13 interacts with both GAB1 and SPROUTY2 to form a tripartite complex.

The GAB1 variant dysregulates MET-signaling pathway genes. GAB1 is an adapter protein for MET, the receptor for HGF (19). We reported that noncoding variants of human HGF cause nonsyndromic hearing loss DFNB39 in many human families (20), and a likely pathogenic variant in $M E T$ is associated with DFNB97linked hearing loss (21). Moreover, deficits of SHP2 and SPRY2 result in hearing loss in humans and mice, respectively (22-24). Therefore, using real-time PCR and lymphoblastoid cell lines generated from the affected and nonpenetrant individuals of family PK-2, we studied the expression of 37 genes related to HGF signaling (Figure 8) $(25,26)$. Among these genes, MET, AKT3, hRAS, STAT1, FAS-R, RAC1, C3G, RAP-1a, GRB2, SHC, EGR1, JAK1, and $E L K 1$ were downregulated by more than 2 -fold $(P<0.05)$ in both DFNB26 deaf and nonpenetrant individuals (Figure 8), whereas PI3K, encoding phospohoinositide 3-kinase, was upregulated $(>2$-fold; $P<0.05)$ in both affected and nonpenetrant individuals (Figure 8). Intriguingly, SPRY2 was significantly $(P<0.001)$ upregulated only in the lymphoblastoid cell lines from the 3 deaf individuals of family PK-2. Taken together, these results indicate a dysregulation of the HGF-signaling pathway among the deaf individuals of family PK-2 who are homozygous for the GAB1 DFNB26 
A

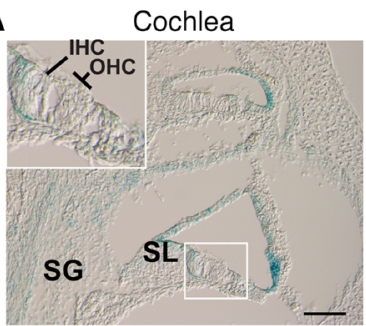

D

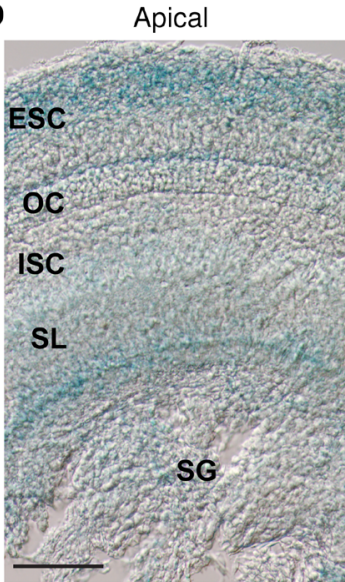

B Ampulla
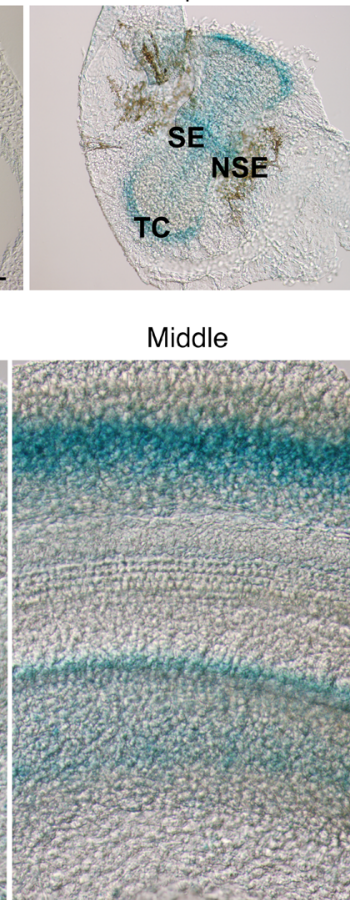

E

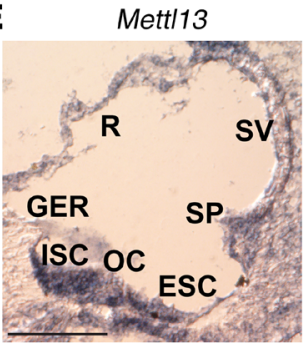

$\mathbf{F}$

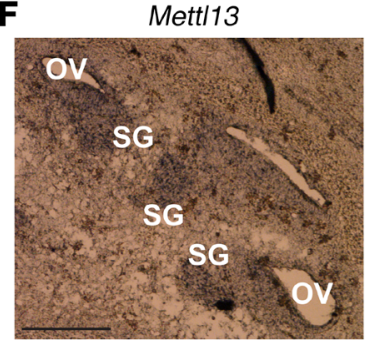

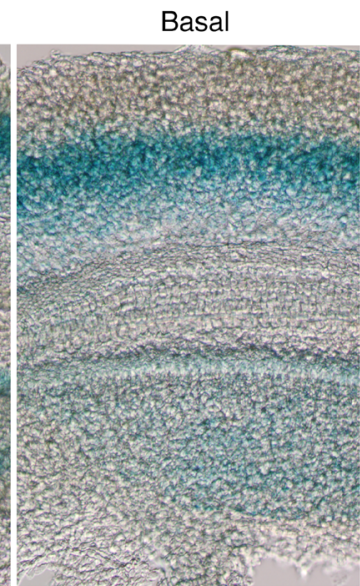

C

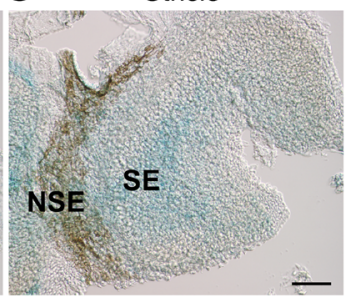

G Negative control

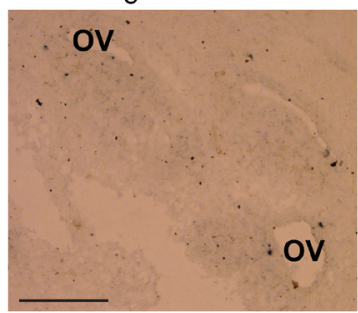

Figure 4. Gab1 and Mett/13 are expressed in mouse inner ear. (A-C) X-gal staining of $\beta$-gal activity as a reporter of Gab1 expression in the cochlear and vestibular epithelia of mouse inner ear. (A) X-gal staining of the middle turn of a cross section of the cochlea of a $\mathrm{Cab1}^{+/}$ mouse at PO shows $\beta$-gal activity in supporting cells of the organ of Corti, spiral ganglion neurons (SG), internal and external sulcus cells, spiral limbus (SL) region, and Reissner's membrane. No detectable staining (see inset) was observed in inner (IHC) or outer (OHC) hair cells. (B and C) $\beta$-Gal activity was observed in the sensory epithelium (SE) and transition cells (TC), while very low or no staining was observed in nonsensory epithelium (NSE). (D) Whole-mount X-gal staining of the apical, middle, and basal turn of cochlea from a Cab1+/- mouse at $\mathrm{PO}$ shows spreading of $\beta$-gal activity in the supporting cells of the organ of Corti (OC), spiral ganglion neurons (SC), internal (ISC) and external (ESC) sulcus cells, and spiral limbus region. (E) At P5, ubiquitous expression of Mett/13 was visible in the region of sensory cells of the cochlea, inner and outer sulcus cells, stria vascularis (SV), Reissner's membrane (R), and spiral ganglion neurons. GER, greater epithelial ridge; SP, spiral prominence. (F) Likewise, in the vestibular tissue on E16, Mett/13 expression was detected in the developing sensory epithelium and ganglion cells. OV, otic vesicle. (G) No expression was found when a Mett/13 sense probe was used as a negative control. Scale bars: $10 \mu \mathrm{m}$; $4 \mu \mathrm{m}$ (inset in $\mathbf{A}$ ). variant. Among the genes examined, only SPRY2 was regulated differently when affected and nonpenetrant individuals homozygous for the GAB1 variant were compared, which points to the mechanism by which the METTL13 variant functions as a modifier to prevent GAB1-associated deafness.

\section{Discussion}

GAB1 is a member of the insulin receptor substrate 1-like (IRS1like) multisubstrate docking adapter protein family. Like other IRS1 adapter proteins, GAB1 has a PH domain that facilitates interaction with phosphatidylinositols within cell membranes and is involved in assembling complexes that function downstream of cell-surface RTKs $(27,28)$. Activated RTK signaling controls a variety of critical cellular processes including cell-cycle progression, differentiation, metabolism, survival, adhesion, motility, and migration. GAB1 is ubiquitously expressed, and in mice, a homozygous deletion of the sequence encoding the $\mathrm{PH}$ domain of GAB1 results in embryonic lethality between E12.5 and E18.5 $(17,29,30)$. Likewise, we found that knockdown of gab1 in zebrafish embryos also resulted in severe developmental abnormalities and lethality in zebrafish embryos (Figure $2 \mathrm{~K}$ ). In contrast, in the PK-2 family, all of the deaf individuals who are homozygous for the p.(Gly116Glu) variant and do not carry the METTL13- dominant modifier variant have prelingual, nonsyndromic severe-to-profound hearing loss (7, 31). Medical and developmental histories, physical examination, and routine blood and urine chemistry tests did not reveal any extra-auditory features in these affected individuals (31). The results of our biochemical lipidbinding assay as well as the zebrafish rescue studies support the assumption that the p.(Gly116Glu) substitution of GAB1 retains a reduced level of function (hypomorphic variant) compared with WT GAB1, which may explain the restricted deafness phenotype segregating in family PK-2.

GAB1 also has a MET receptor-binding site, a central prolinerich domain, and several tyrosyl and seryl/threonyl phosphorylation sites. The PH domain of GAB1 has a role in the recruitment of B cell antigen receptors and can act as a signal amplifier (32). Pathogenic variants in $\mathrm{PH}$ domains of various other proteins cause the human disorders $\mathrm{X}$-linked agammaglobulinemia (OMIM 300755) $(33,34)$ and X-linked immunodeficiency in mice $(35,36)$. Likewise, variants in the $\mathrm{PH}$ domain of dynamin 2 are associated with Charcot-Marie-Tooth disease (OMIM 606482) (37). In our study of hereditary deafness, we provide evidence that a missense variant (c.347G>A (p.Gly116Glu)) in the PH domain of human GAB1 causes nonsyndromic, profound, prelingual, sensorineural deafness DFNB26. 


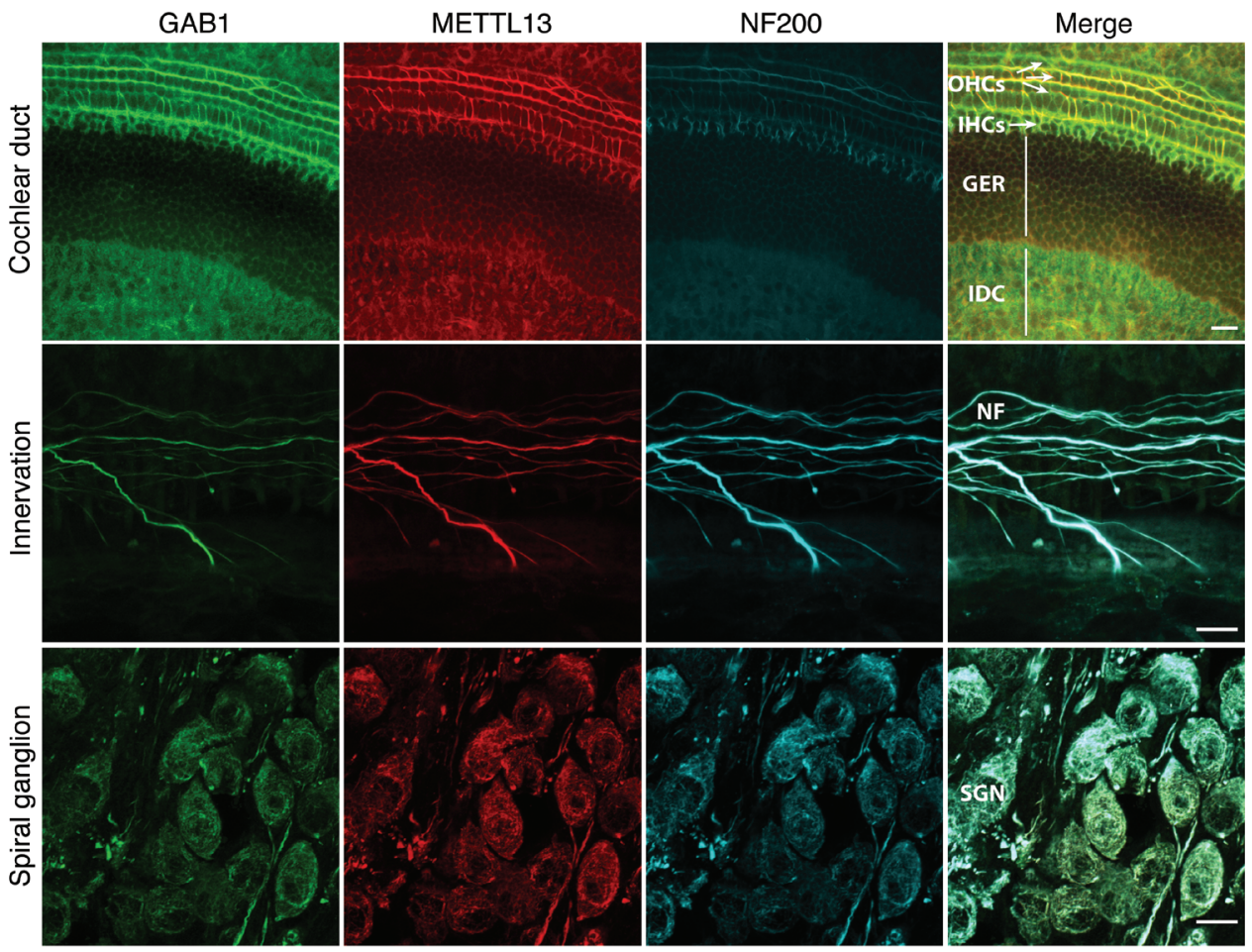

Figure 5. GAB1 and METTL13 colocalize in sensory neurons. Triple labeling of mouse cochlear whole mounts with antibodies against GAB1 (green), METTL13 (red), and the neurofilament marker NF200 (cyan) show colocalization in the nerve fibers that innervate $\mathrm{IHCs}$ and $\mathrm{OHCs}$ in the cochlear duct (top). Magnified images of neuronal processes (NF) running parallel to the organ of Corti (middle) is shown. Spiral ganglion (bottom) revealed colocalization of GAB1, METTL13, and NF200 in the neuronal cell bodies (SCN) as well. IDC, interdental cells. Scale bars: $10 \mu \mathrm{m}$.

In addition to GAB1 functions downstream of HGF via the MET receptor (30), GAB1 interacts with other proteins that are essential for inner ear development and function. GAB1 transmits signals from IGF-1 receptors to ERK via SH2-containing protein tyrosine phosphatase (SHP2) (38). In humans, a homozygous deletion of the IGF1 gene causes severe sensorineural hearing loss, growth failure, and mental retardation (OMIM 608747) (39). Igf1 $1^{-/-}$mutant mice have altered innervation of the organ of Corti and have defects in the postnatal survival, differentiation, and maturation of cochlear ganglion cells $(40,41)$. GAB1 also functions downstream of erythroblastic leukemia viral oncogene homolog 2 (ErbB2), a member of the EGF family of receptors that is activated upon stimulation by neuregulins (NRGs) (42-44). Transgenic mice with dominant-negative ErbB receptors have degeneration of cochlear neurons and severe hearing loss (45). SHP2 and PI3K (30) are also prominent effectors of GAB1. Pathogenic variants in SHP2 result in Noonan (OMIM 163950) and LEOPARD syndromes (OMIM 151100) with multiple overlapping features including hearing loss (46). SPROUTY2, encoded by Spry2, interacts directly with GRB2 (47), and loss of its function also causes hearing loss in mice (23). Interestingly, many of these genes, including $S P R Y 2$, are dysregulated in the lymphoblastoid cell lines derived from the individuals homozygous for the DFNB26 GAB1 missense variant, which we hypothesize affects 1 or more of the downstream signaling pathways leading to altered cell survival, apoptosis, cell migration, and cell adhesion (Supplemental Figure 7). Additional model systems will be required to understand in greater detail the role of the GAB1-mediated signaling pathway that is essential for normal hearing.

Modifiers in the genetic background that enhance or suppress a Mendelian disorder provide insight into the mechanisms by which organisms can buffer biological processes to accommodate the adverse effects of pathogenic genetic variants (48-50), point- ing toward possible therapies for human disorders. We discovered that a variant of METTL13 (DFNM1) completely suppressed DFNB26 hearing loss associated with a $G A B 1$ missense variant. In zebrafish, human METTL13 harboring the c.1634G $>$ A modifier variant was able to significantly suppress the morphant phenotype. NanoSPD and co-IP studies revealed that there is either an indirect interaction through an adaptor or a direct interaction between METTL13, GAB1, and SPRY2 proteins. The protein expression and distribution of GAB1 and METTL13 share similarities with other proteins, including IGF-1, ErbB2, proteolipid protein 1 (PLP), and nerve growth factor (NGF) $(40,41,51-53)$. It is possible, therefore, that GAB1 and METTL13 proteins are components of an inner ear signaling pathway that involves 1 or more of these proteins. METTL13 is located within the meiotic boundaries of the dominantly inherited hearing loss locus DFNA7 (OMIM 601412), for which a pathogenic variant has not been reported (54). Given the SPRY2 expression rescue and interaction with SPROUTY2, we also speculate that the modifier allele of METTL13 may reduce the severity of sensorineural hearing loss caused by pathogenic variants of SPRY2, MET, or HGF.

METTL13 is a functionally uncharacterized protein that is overexpressed in some human cancers. However, it has low expression in normal tissues including the testis, brain, and liver (55). In transgenic mice, Mettl13 ectopic overexpression can drive tumorigenesis in vivo (55). Moreover, gene expression profiling of NIH3T3 cells overexpressing METTL13 dysregulated the RTK and hedgehogsignaling pathways (55). Intriguingly, METTL13 is predicted to have methyltransferase activity, spermidine/spermine synthase activity, or ubiquinone synthase activity, but these functions of METLL13 have not been experimentally demonstrated (55).

In summary, through genetic linkage analyses, positional cloning, and massively parallel sequencing, we have identified 

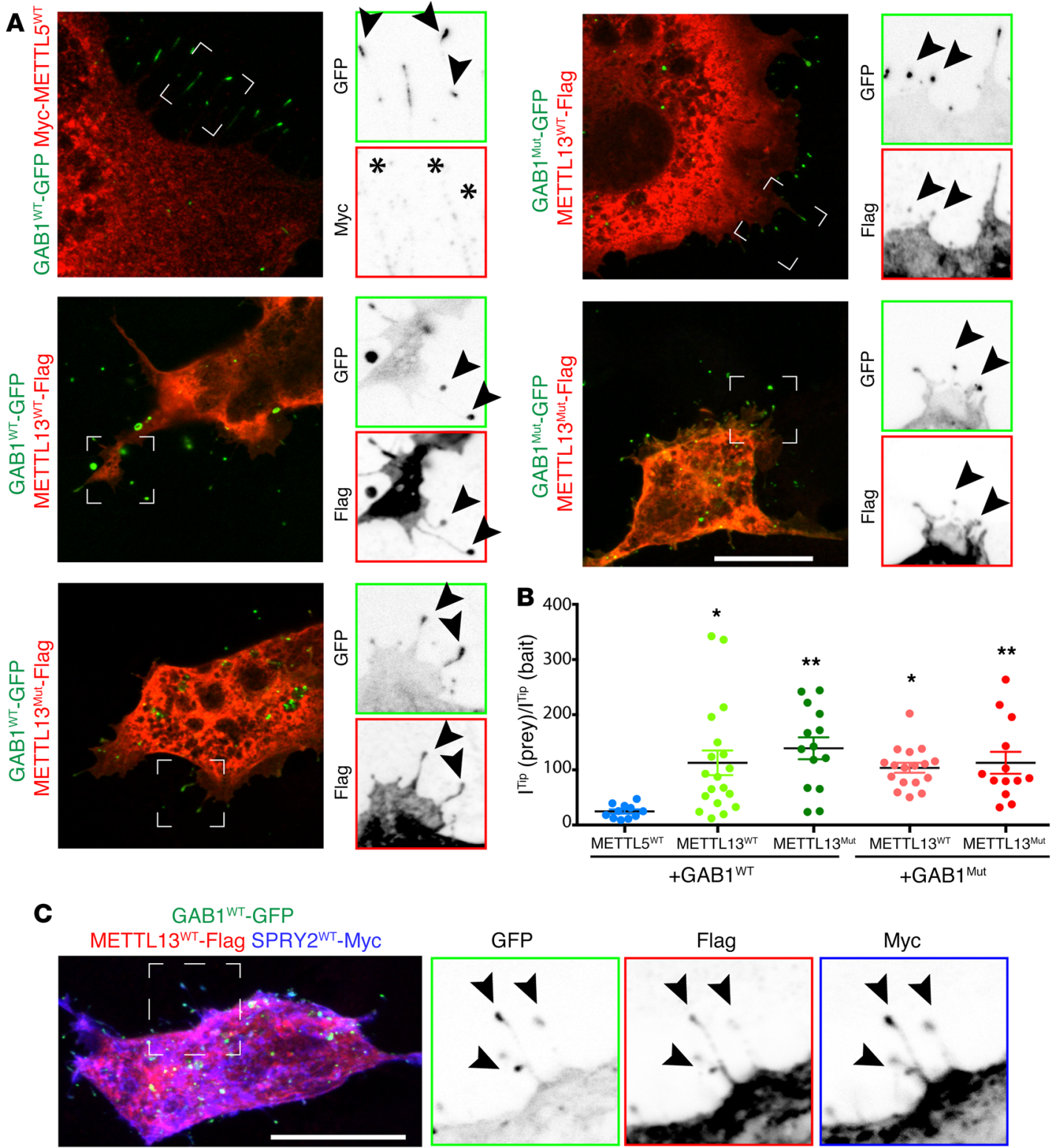

Figure 6. GAB1, METTL13 and SPROUTY2 form a tripartite complex at filopodia tips. (A) COS-7 cells were cotransfected with the nonfluorescent

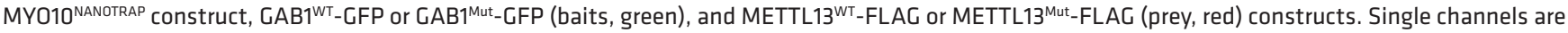
shown as inverted grayscale images. Accumulations at the tip of the bait and prey are shown with arrowheads. Asterisks indicate the absence of prey accumulation at the filopodia tip. A Myc-METTL5 ${ }^{\mathrm{WT}}$ expression construct was used as a negative control. (B) Quantification of fluorescence intensities revealed significant accumulation of GAB-GFP and METTL13-Flag proteins at filopodia tips. ${ }^{*} P<0.05$ and ${ }^{* *} P<0.01$, by 1-way ANOVA. Data represent the mean \pm SEM. (C) COS-7 cells were cotransfected with nonfluorescent MY010 ${ }^{\text {NANOTRAP }}$, GAB1WT-GFP (bait, green), METTL13 ${ }^{\text {WT }}$-Flag (red), and SPRY2 ${ }^{\text {WT }}$-Myc (blue) constructs. Single channels are shown as inverted grayscale images. Both bait and prey accumulated together at the tips of the filopodia (arrowheads), indicating an interaction between the bait and prey. Scale bars: $10 \mu \mathrm{m} ; 5 \mu \mathrm{m}$ (enlarged panels in $\mathbf{A}$ and $\mathbf{C}$ ).

a missense variant in human GAB1 cosegregating with DFNB26 hearing loss. A dominant allele in a predicted methyltransferase gene, METTL13, entirely suppresses human deafness associated with a $G A B 1$ variant. Our zebrafish in vivo data support a modifying role of suppressor human METTL13 mRNA to partially rescue the phenotype associated with the human GAB1-mutant allele. Pulldown studies revealed a tripartite complex between METTL13, GAB1, and SPROUTY2. Among the HGF-signaling pathway genes tested, only SPRY2 was differentially regulated between affected and nonpenetrants, which could be the mechanism by which the METTL13-dominant variant functions as a modifier to prevent GAB1-associated deafness. MET/HGF signaling is essential for diverse functions in many tissues besides inner ear, therefore, an understanding of the mechanism of METTL13-based modification will likely have broader clinical implications.

\section{Methods}

Subject and clinical evaluations. Family ascertainment, clinical evaluations including hearing and vestibular function testing, and genomic DNA extraction were previously described (7). 


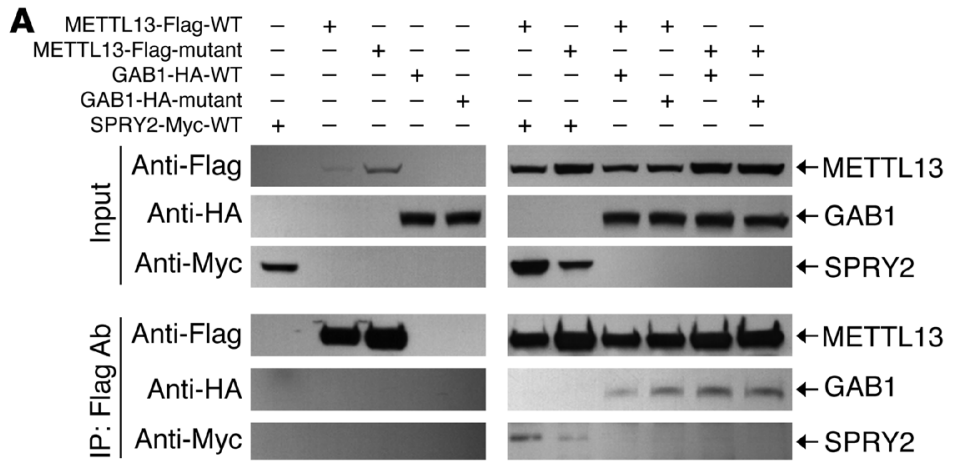

B

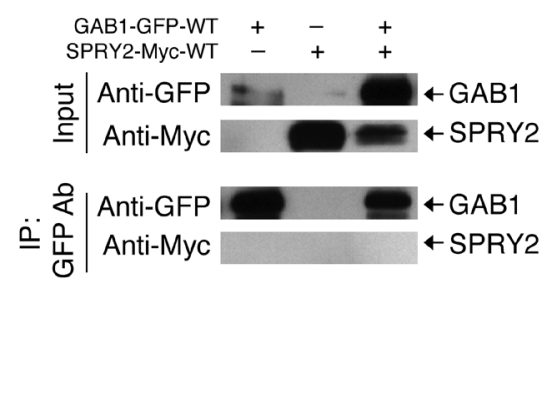

Figure 7. METTL13 interacts with GAB1 and SPROUTY2. (A) Lysates from HEK293 cells transfected with both normal and mutant METTL13-Flag- along with normal and mutant CAB1-HA- and Myc-tagged SPRY2 expression constructs in various combinations were used for co-IP assays with anti-Flag antibody-coated beads. Precipitates were immunoblotted with antibodies against Flag, HA, and Myc tags. (B) Co-IP assay shows an absence of interaction between GAB1 ${ }^{\text {WT }}$-GFP and SPRY2 ${ }^{\text {WT }}$-Myc.

Candidate gene screening. Genes within the DFNB26 and DFNM1 chromosomal intervals and the exomes in the 2 linkage intervals were screened for rare variants. Sanger sequencing was performed on PCR amplimers of genomic DNA obtained from 2 deaf individuals, 1 normalhearing nonpenetrant individual, and 1 normal-hearing individual WT at the DFNB26 and DFNM1 loci, all of whom are members of the PK-2 family. Primers were designed with Primer3 software (http:// frodo.wi.mit.edu/primer3) for amplification of all exons of each candidate gene, including the intron-exon boundaries. Methods for direct sequencing and mutational analyses were described previously (56). BigDye terminator reaction products were resolved on Applied Biosystems 377 or 3730 instruments. Sequencing traces were analyzed with SeqMan software (DNASTAR, Lasergene).

Segregation of GAB1 and METTL13 alleles in the PK-2 family was confirmed by direct sequencing of PCR products amplified from genomic DNA from all participating family members. Genomic DNA samples from control, presumably normal-hearing individuals from Pakistan and India and from a human diversity panel (cell repositories, Coriell Institute for Medical Research) were also analyzed for variants.

Exome sequencing. Genomic DNA samples from an affected individual, a nonpenetrant individual, and a normal-hearing individual were sheared to fragments of approximately $200 \mathrm{bp}$ and used to make libraries for paired-end sequencing (Illumina). The libraries were hybridized to biotinylated cRNA oligonucleotide probes from the SeqCap EZ Exome Library v2.0 (NimbleGen), purified by streptavidinbound magnetic beads, amplified, and sequenced using an Illumina HiSeq 2000 Analyzer. We generated a total of $37 \mathrm{~Gb}$ of sequence as paired-end, 100-bp reads from 1 lane of an Illumina HiSeq 2000 Analyzer. For sequence alignment, analysis, and variant identification, we used the Broad Institute's Genome Analysis Toolkit (GATK) $(57,58)$.

Zebrafish embryo manipulation, $M O$ injection, and expression profiling. Anti-gab1 translation-blocking (5'-AGTAAACCACATCTCCTCCGCTCAT-3'), splice-site (5'-TCCATGCCTGAAAACAGAAACAACA-3'), and controlMO (5'-GGAAAAGACCGCTTTAAACTTTCGT-3') oligonucleotides (Gene Tools) were diluted to $2.5 \mathrm{ng}$, $5.0 \mathrm{ng}$, and $7.5 \mathrm{ng}$ in deionized, sterile water for dose-response experiments and $2.5 \mathrm{ng}$ for rescue experiments, and then injected into WT zebrafish embryos at the 1- or 2-cell stage. To rescue morphant phenotypes, we transcribed human GAB1 and METTL13 mRNA from a linearized pT7TS vector with the T7 mMESSAGE mMACHINE Kit (Ambion, Thermo Fisher Scientific). A total of 1,000 pg and $250 \mathrm{pg}$ GAB1 and METTL13 mRNA, respectively, were coinjected along with the Gab1 MO into WT zebrafish embryos.

For zebrafish gab1 and mettl13 expression profiling at various developmental stages, total RNA was extracted using a RiboPure Kit (Ambion, Thermo Fisher Scientific) from 18 to 20 zebrafish embryos for each time point (0 hour to 5 days). RNA was reverse transcribed using SuperScript RT (Clontech). Real-time quantitative RT-PCR analyses were performed using the StepOne Plus Real-Time System (Applied Biosystems) with RT2 ${ }^{2}$ SYBR Green qPCR Mastermix (QIAGEN). The relative expression values were normalized to an endogenous gapdh control (Supplemental Table 5).

For temporal expression of $g a b 1$ and mettl13 in zebrafish, RNA ISH at different developmental stages from 1 day post fertilization (dpf) to $5 \mathrm{dpf}$ was performed as previously described using a standard procedure (59). A partial sequence of approximately $600 \mathrm{bp}$ of C-terminal cDNA of gab1 and mettl13 was used to generate digoxigenin-labeled sense and antisense RNA probes. A sense probe was used in each experiment as a control and gave no hybridization signal.

PH domain phospholipid-binding assay. GST fusion proteins were generated by subcloning into a pGEX-5.1 vector (Amersham Pharmacia Biotech). GAB1 cDNA fragments encoding the PH domain (amino acid residues 24-120; NP_997006) with and without the p.Gly116Glu missense variant were cloned and expressed in E. coli BL21 (Stratagene). GST fusion proteins were purified on glutathione-sepharose using a GST Bulk Kit according to the manufacturer's instructions (Amersham Pharmacia Biotech). Equal amounts of soluble WT and mutant GSTPH domain fusion proteins $(0.5 \mu \mathrm{g} / \mathrm{ml})$ were incubated with PIP Array Membranes (P-23748; Invitrogen, Thermo Fisher Scientific) for 4 hours, followed by washing, and then probed with anti-GST antibody. The experiment was replicated 3 times using fresh preparations of WT and mutant proteins and new PIP arrays. Signal intensities at the lipid spots were normalized against the blot background intensity and then against PI(5)P lipid-binding signals from the WT GAB1-PH domain protein before plotting.

$\beta$-Gal staining. A Gab1-knockout mouse has exon 2 encoding part of the PH domain (amino acids 25-123; AAH94659) replaced with a promoter-less neomycin resistance gene, an internal ribosome entry 

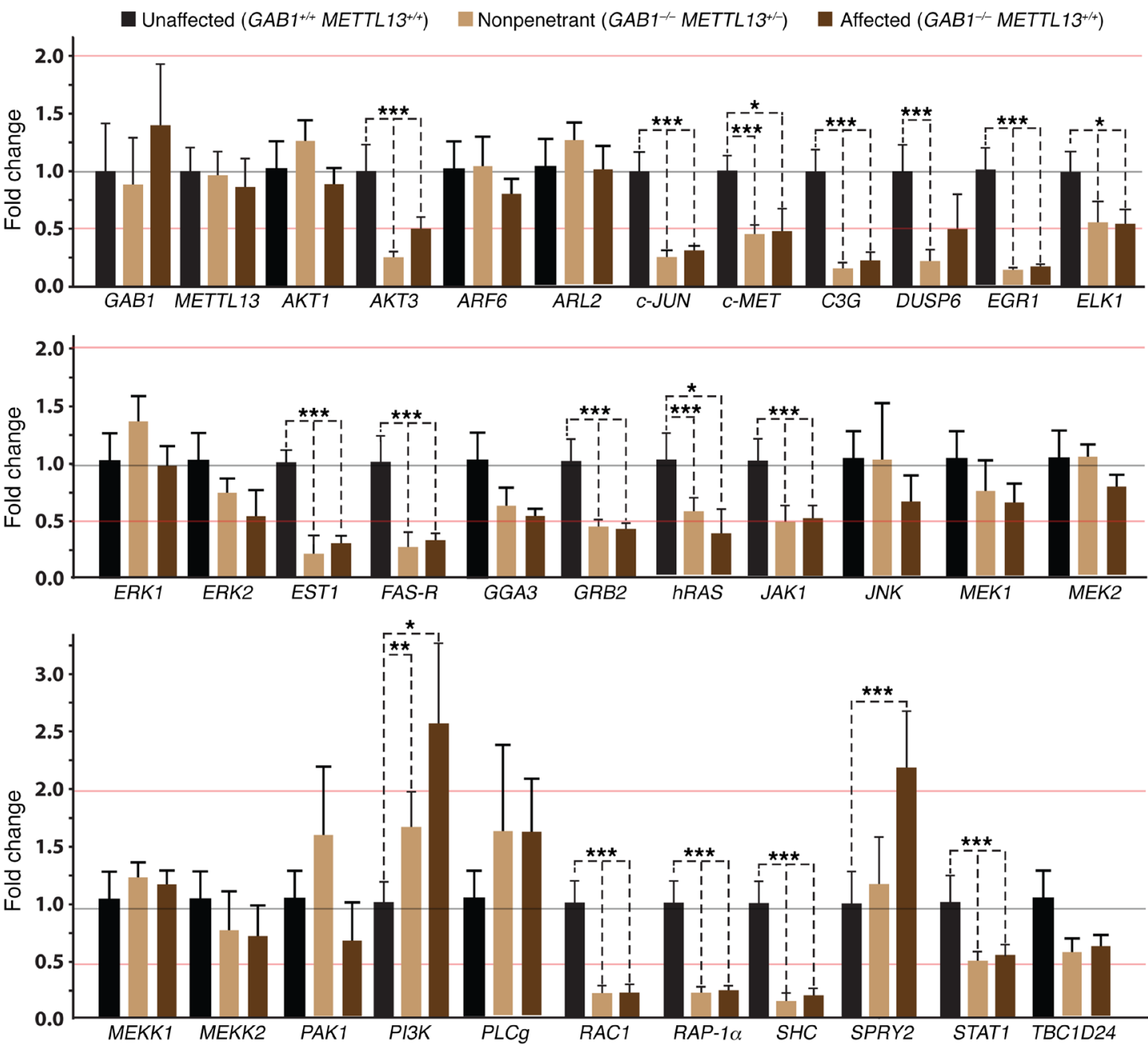

Figure 8. MET-signaling pathway genes are dysregulated in the lymphoblastoid cell lines of individuals homozygous for the GAB1 DFNB26 deafnessassociated variant. Real-time PCR was performed on cDNA generated from lymphoblastoid cell lines from 3 affected individuals and 2 nonpenetrant individuals of family PK-2 and from a normal-hearing control sample. Shown are data for the genes dysregulated in individuals homozygous for the c.347C $>$ A (p.Gly116Glu) DFNB26 variant of GAB1. ${ }^{*} P<0.05,{ }^{* *} P<0.01$, and ${ }^{* * *} P<0.001$, by 1 -way ANOVA. Data represent the mean \pm SEM. In contrast to deaf individuals, $S P R Y 2$ expression is normal in nonpenetrant individuals.

site (IRES), and the LacZ reporter gene (17). To determine expression, we used an important feature of the targeting cassette. Expression of the $L a c Z$ reporter gene is under the control of the endogenous Gab1 promoter (17). Whole cochleae were isolated from P5 $\mathrm{Gab1}^{+/-}$-knockout mice (17) and fixed for 20 minutes in $1 \%$ formaldehyde, $0.2 \%$ gluteraldehyde, and $0.02 \% \mathrm{NP}-40$ in $1 \times \mathrm{PBS}$, washed twice with solution containing $2 \mathrm{mM} \mathrm{MgCl}_{2}$ and $0.02 \% \mathrm{NP}-40$ in $1 \times \mathrm{PBS}$, and then incubated overnight in the dark at $37^{\circ} \mathrm{C}$ in $\mathrm{X}-\mathrm{Gal}$ staining solution $(1 \mathrm{mg} / \mathrm{ml}$ $\mathrm{X}$-Gal, $5 \mathrm{mM}$ potassium ferrocyanide, $5 \mathrm{mM}$ potassium ferricyanide, and $0.1 \mathrm{M} \mathrm{MgCl} 2$, in $1 \times \mathrm{PBS}$ ). Cochleae were dissected, and the organ of Corti, ampules, utricle, and saccule were isolated, mounted onto a slide, and observed using a Zeiss LSM700 equipped with DIC optics.

ISH studies. Frozen sections of mouse hemidissected heads (E11.5, E15.5, and P5) were processed for ISH essentially as described previously (60). All samples were permeabilized with proteinase $\mathrm{K}$, dehydrated in $30 \%$ sucrose, embedded in gelatin, and sectioned. A fulllength mouse Mettl13 cDNA (NM_144877), including approximately $1 \mathrm{~kb}$ of the $3^{\prime}$-UTR, was used to generate digoxigenin-labeled sense and antisense RNA probes. A sense probe was used in each experiment as a control and gave no hybridization signal.

Antibodies and immunofluorescence staining. GAB1 (PB296) and METTL13 (PB477, PB448) antisera were raised in rabbits against protein-specific peptides (SLEGFHSQYKIKSVL, corresponding to residues 420-434 of the mouse GAB1 sequence [NP_067331.2], and KDTSHRAQKKRKKDRK, corresponding to residues 431-446 of the mouse METTL13 sequence [NP_659126.1], respectively). Antisera were affinity purified using a Pierce Protein A IgG Purification Kit (Thermo Fisher Scientific), and the synthetic peptide that was used as the immunogen.

For immunofluorescence staining, cochleae were isolated from WT C57BL/ 6 mice and fixed in $4 \%$ paraformaldehyde overnight, washed with PBS for a few minutes, and then dissected to extract the organ of Corti and vestibular epithelia, which were washed 3 times with $1 \times$ PBS for 10 minutes each and permeabilized with $0.2 \%$ Triton $\mathrm{X}-100$ diluted in $1 \times$ PBS for 30 minutes at room temperature. Tissues were incubated in blocking solution ( $5 \%$ BSA and $2 \%$ goat serum) at room temperature for 1 hour and then incubated for 2 hours with pri- 
mary antibody diluted at 1:200 in blocking solution. The tissues were then washed with PBS and stained with an anti-rabbit Alexa Fluor 488 IgG (Invitrogen, Thermo Fisher Scientific) for 20 minutes at room temperature. Samples were mounted with ProLong Gold Reagent and imaged using an LSM710 confocal microscope equipped with a $\times 63$ 1.4 numerical aperture (NA) objective (Zeiss Microimaging).

Antibody specificity was validated by transfections (Lipofectamine 2000; Life Technologies, Thermo Fisher Scientific) of GFP-tagged GAB1 or METTL13 into COS-7 cells, followed by immunostaining with PB296 and PB447 antisera, respectively. Full-length human GAB1 (NM_207123) and METTL13 (NM_015935) cDNAs were subcloned into a pEGFP-N2 vector (Clontech). COS-7 cells were cultured in $10 \%$ FBS-supplemented DMEM. Fluorescence immunocytochemistry was performed on fixed cells (4\% paraformaldehyde, for $30 \mathrm{~min}$ ).

Nanoscale pulldown assay. Nanoscale pulldown (NanoSPD) assays were performed using the MYO10 ${ }^{\text {NANOTRAP }}$ construct as described previously (18). Briefly, COS-7 cells were cultured in DMEM medium supplemented with $10 \% \mathrm{FBS}$ and incubated at $37^{\circ} \mathrm{C}$ in $5 \% \mathrm{CO}_{2}$. Cell transfections were performed using the Lipofectamine 2000 protocol (Thermo Fisher Scientific). A NanoSPD assay was performed using COS-7 cells cotransfected with $1 \mu \mathrm{g}$ MYO10 ${ }^{\text {NANOTRAP }}-$, GAB1-GFP-, METTL5-Myc-, METTL13-Flag-, or SPRY2-Myc-tagged constructs. Twenty-four hours after transfection, cells were trypsinized and transferred onto 3.5-mm collagen-coated, glass-bottomed culture dishes (MatTek Corporation). Cells were incubated for another 24 hours and then fixed with $4 \%$ paraformaldehyde for 20 minutes. Finally, transfected cells were processed for the immunostaining protocol and imaged using a confocal microscope. Quantification of fluorescence intensities at the tips of the filopodia was performed using Image J software (NIH). Each value is an average over a square area of $1 \mu \mathrm{m}^{2}$, with a center at the tip of each individual filopodium $(n \geq 15)$. Data are expressed as a ratio of Alexa Fluor 546 fluorescence/GFP intensities. Each value was then normalized against the values measured in the cells transfected with the bait constructs only.

Co-IP assay. Flag-tagged normal METTL13 and mutant METTL13 under the control of CMV promotor stable cell line were generated in HEK293 cells and maintained using DMEM supplemented with 10\% FBS, glutamine, and penicillin-streptomycin (Invitrogen, Thermo Fisher Scientific). Cells were plated at $80 \%$ confluency for 24 hours at $37^{\circ} \mathrm{C}$ in $5 \% \mathrm{CO}_{2}$. On the day of transfection, $10 \mu \mathrm{g}$ of each DNA was transfected into cells using $1 \mathrm{mg} / \mathrm{ml}$ Poly(ethyleneimine) (PEI) (MilliporeSigma). After 48 hours, cells were harvested and disrupted with a sonicator (Thermo Fisher Scientific) at intensity setting 2, for three 10-second pulses in buffer A (50 mM Tris- $\mathrm{HCl}, \mathrm{pH} 7.5,100 \mathrm{mM} \mathrm{NaCl}$, and 1\% NP-40) containing a protease inhibitor cocktail (MilliporeSigma). Anti-Flag M2 Affinity Agarose Gel (MilliporeSigma) was incubated with lysates for 16 hours and then centrifuged at 10,000 $g$ for 3 minutes. Beads were washed with buffer A 3 times and boiled in $2 \times$ SDS sample buffer (Invitrogen, Thermo Fisher Scientific). The samples were run on $4 \%$ to $20 \%$ SDS PAGE Gels (Invitrogen, Thermo Fisher Scientific) and probed with anti-Flag, anti-HA, anti-Myc, or anti-ELMOD3 antibodies to detect Myctagged METTL13, GAB1, SPRY2, and ELMOD3, respectively.

Gene expression profiling using semiquantitative real-time PCR. Total RNA was isolated from 6 different lymphoblastoid cell lines that were derived from EBV-transformed lymphocytes. Lymphocytes were isolated from blood samples from 3 affected individuals, 2 nonpenetrant individuals, and 1 normal-hearing individual of family PK-2. TRIzol
(Invitrogen, Thermo Fisher Scientific) was used to extract RNA. cDNA was synthesized with a SMART First-Strand cDNA Synthesis Kit (Clontech) and oligo-dT primers. Subsequent PCR reactions were performed with gene-specific primers and TaqMan (Applied Biosystems) or SYBR Green probes (Supplemental Table 5). TaqMan probes were designed to span exon-to-exon splice junctions. Semiquantitative real-time PCR was performed in triplicate on a StepOnePlus PCR System (Applied Biosystems). Probes targeting human GAPDH, EUK $18 \mathrm{~S}$ rRNA, $\beta$-actin, and/or $\beta 2$ microglobin (Applied Biosystems) were used as endogenous controls.

Statistics. In vitro binding assays, co-IP studies, zebrafish rescue assays, and real-time PCR experiments were repeated at least 3 times. All data represent the mean \pm SEM. One-way ANOVA with Dunnett's test was used to compare the different groups of independent samples. Statistical significance in lipid binding ability was determined using 2-tailed Student's $t$ test. The level of significance was set at 0.05 for all statistical tests.

Study approval. This study was approved by the IRBs of the National Centre of Excellence in Molecular Biology (NCEMB) in Lahore, Pakistan (FWA00001758), the NIH (Combined Neuroscience Blue Panel IRB; OH-93-N-016), and the University of Maryland School of Medicine (HP-00061036). Written informed consent was obtained from adult subjects and parents of minor subjects.

\section{Author contributions}

$\mathrm{RY}$ and ZMA designed and performed research, analyzed data, and wrote the manuscript. APJG, AL, and AD designed and conducted research. RJM and ERW analyzed data. Sheikh Riazuddin, TBF, and Saima Riazuddin designed the research project, acquired and analyzed data, provided reagents, and wrote the manuscript.

\section{Acknowledgments}

We thank Doris Wu at NIDCD, Saulius Sumanas and Gowri Nayak at CCHMC, Tingfang Chen, Assad Usmani, and Elodie Richard at UMSOM for their technical assistance, helpful suggestions, and discussion of the results. This research was supported in part by the Intramural Research Program of the NIH, NIDCD (DC000039-20, to TBF), by an Action on Hearing Loss grant, and by NIH, NIDCD research grants R56DC011803 (to Saima Riazuddin) and R01DC012564 to (ZMA).

Address correspondence to: Saima Riazuddin, Laboratory of Molecular Genetics, Department of Otorhinolaryngology - Head and Neck Surgery, University of Maryland School of Medicine, 800 West Baltimore St., Room 404, Baltimore, Maryland, 21201. Phone: 410.736.3798; Email: sriazuddin@som.umaryland.edu.

RY's present address is: Baylor College of Medicine, Houston, Texas, USA.

RJM's present address is: Genomic \& Computational Biology Core, NIDCD, NIH, Bethesda, Maryland, USA.

AD's present address is: Sunnybrook Research Institute, Sunnybrook Health Sciences Centre, Toronto, Ontario, Canada.

ERW's present address is: DNA Sequencing Center, Brigham Young University, Provo, Utah, USA. 
1. Hokanson JE. Functional variants in the lipoprotein lipase gene and risk cardiovascular disease. Curr Opin Lipidol. 1999;10(5):393-399.

2. Mucci LA, Wedren S, Tamimi RM, Trichopoulos $\mathrm{D}$, Adami HO. The role of gene-environment interaction in the aetiology of human cancer: examples from cancers of the large bowel, lung and breast. J Intern Med.2001;249(6):477-493.

3. Whalley LJ. Early-onset Alzheimer's disease in Scotland: environmental and familial factors. $\mathrm{BrJ}$ Psychiatry Suppl. 2001;40:s53-s59.

4. Zielenski J. Genotype and phenotype in cystic fibrosis. Respiration. 2000;67(2):117-133.

5. Marchani EE, et al. Evidence for three loci modifying age-at-onset of Alzheimer's disease in early-onset PSEN2 families. Am J Med Genet $B$ Neuropsychiatr Genet. 2010;153B(5):1031-1041.

6. Guan MX, et al. Mutation in TRMU related to transfer RNA modification modulates the phenotypic expression of the deafness-associated mitochondrial $12 \mathrm{~S}$ ribosomal RNA mutations. $\mathrm{Am}$ JHum Genet. 2006;79(2):291-302.

7. Riazuddin S, et al. Dominant modifier DFNM1 suppresses recessive deafness DFNB26. Nat Genet. 2000;26(4):431-434.

8. Bykhovskaya Y, et al. Candidate locus for a nuclear modifier gene for maternally inherited deafness. Am J Hum Genet. 2000;66(6):1905-1910.

9. Schultz JM, et al. Modification of human hearing loss by plasma-membrane calcium pump PMCA2. N Engl J Med. 2005;352(15):1557-1564.

10. Estivill $X$, et al. Familial progressive sensorineural deafness is mainly due to the mtDNA A1555G mutation and is enhanced by treatment of aminoglycosides. Am J Hum Genet. 1998;62(1):27-35.

11. Young WY, et al. Extremely low penetrance of hearing loss in four Chinese families with the mitochondrial 12S rRNA A1555G mutation. Biochem Biophys Res Commun. 2005;328(4):1244-1251.

12. 1000 Genomes Project Consortium, et al. A global reference for human genetic variation. Nature. 2015;526(7571):68-74

13. Fu W, et al. Analysis of 6,515 exomes reveals the recent origin of most human protein-coding variants. Nature. 2013;493(7431):216-220.

14. Lek M, et al. Analysis of protein-coding genetic variation in 60,706 humans. Nature. 2016;536(7616):285-291.

15. Rehman AU, et al. Challenges and solutions for gene identification in the presence of familial locus heterogeneity. Eur J Hum Genet. 2015;23(9):1207-1215.

16. Bill BR, Petzold AM, Clark KJ, Schimmenti LA, Ekker SC. A primer for morpholino use in zebrafish.Zebrafish. 2009;6(1):69-77.

17. Itoh M, Yoshida Y, Nishida K, Narimatsu M, Hibi M, Hirano T. Role of Gab1 in heart, placenta, and skin development and growth factor- and cytokine-induced extracellular signal-regulated kinase mitogen-activated protein kinase activation. Mol Cell Biol. 2000;20(10):3695-3704.

18. Bird JE, et al. Harnessing molecular motors for nanoscale pulldown in live cells. Mol Biol Cell. 2017;28(3):463-475.

19. Schaeper U, Gehring NH, Fuchs KP, Sachs M, Kempkes B, Birchmeier W. Coupling of Gab1 to c-Met, Grb2, and Shp2 mediates biological responses. JCell Biol. 2000;149(7):1419-1432.
20. Schultz JM, et al. Noncoding mutations of HGF are associated with nonsyndromic hearing loss, DFNB39. Am J Hum Genet. 2009;85(1):25-39.

21. Mujtaba G, Schultz JM, Imtiaz A, Morell RJ, Friedman TB, Naz S. A mutation of MET, encoding hepatocyte growth factor receptor, is associated with human DFNB97 hearing loss. JMed Genet. 2015;52(8):548-552.

22. Derbent $\mathrm{M}$, et al. Clinical and hematologic findings in Noonan syndrome patients with PTPN11 gene mutations. Am JMed Genet A. 2010;152A(11):2768-2774.

23. Shim K, Minowada G, Coling DE, Martin GR. Sprouty2, a mouse deafness gene, regulates cell fate decisions in the auditory sensory epithelium by antagonizing FGF signaling. Dev Cell. 2005;8(4):553-564.

24. Capute AJ, Rimoin DL, Konigsmark BW, Esterly NB, Richardson F. Congenital deafness and multiple lentigines. A report of cases in a mother and daughter. Arch Dermatol. 1969;100(2):207-213.

25. Taketomi T, et al. Loss of mammalian Sprouty2 leads to enteric neuronal hyperplasia and esophageal achalasia. Nat Neurosci. 2005;8(7):855-857.

26. Li C, Scott DA, Hatch E, Tian X, Mansour SL. Dusp6 (Mkp3) is a negative feedback regulator of FGF-stimulated ERK signaling during mouse development. Development. 2007;134(1):167-176.

27. Gu H, Neel BG. The "Gab" in signal transduction. Trends Cell Biol. 2003;13(3):122-130.

28. Liu Y, Rohrschneider LR. The gift of Gab. FEBS Lett. 2002;515(1-3):1-7.

29. Sachs M, et al. Essential role of Gab1 for signaling by the c-Met receptor in vivo.J Cell Biol. 2000;150(6):1375-1384.

30. Schaeper U, Vogel R, Chmielowiec J, Huelsken J, Rosario M, Birchmeier W. Distinct requirements for Gab1 in Met and EGF receptor signaling in vivo. Proc Natl Acad Sci U S A. 2007;104(39):15376-15381.

31. Riazuddin S, Ahmed ZM, Friedman TB, Griffith AJ, Riazuddin S, Wilcox ER. Genetic modifiers of hereditary hearing loss. Adv Otorhinolaryngol. 2002;61:224-229.

32. Cable J, Steel KP. Combined cochleo-saccular and neuroepithelial abnormalities in the Varitint-waddler-J (VaJ) mouse. Hear Res. 1998;123(1-2):125-136

33. Tsukada S, et al. Deficient expression of a B cell cytoplasmic tyrosine kinase in human X-linked agammaglobulinemia. Cell. 1993;72(2):279-290.

34. Vetrie $\mathrm{D}$, et al. The gene involved in $\mathrm{X}$-linked agammaglobulinaemia is a member of the src family of protein-tyrosine kinases. Nature. 1993;361(6409):226-233.

35. Rawlings DJ, et al. Mutation of unique region of Bruton's tyrosine kinase in immunodeficient XID mice. Science. 1993;261(5119):358-361.

36. Thomas JD, Sideras P, Smith CI, Vorechovský I, Chapman V, Paul WE. Colocalization of X-linked agammaglobulinemia and $\mathrm{X}$-linked immunodeficiency genes. Science. 1993;261(5119):355-358.

37. Züchner S, et al. Mutations in the pleckstrin homology domain of dynamin 2 cause dominant intermediate Charcot-Marie-Tooth disease. Nat Genet. 2005;37(3):289-294.

38. Winnay JN, Brüning JC, Burks DJ, Kahn CR. Gab-1-mediated IGF-1 signaling in IRS-1-deficient 3T3 fibroblasts. J Biol Chem.
2000;275(14):10545-10550.

39. Woods KA, Camacho-Hübner C, Savage MO, Clark AJ. Intrauterine growth retardation and postnatal growth failure associated with deletion of the insulin-like growth factor I gene. $N$ EnglJ Med.1996;335(18):1363-1367.

40. Camarero G, et al. Delayed inner ear maturation and neuronal loss in postnatal Igf-1-deficient mice. J Neurosci. 2001;21(19):7630-7641.

41. Camarero G, et al. Cochlear abnormalities in insulin-like growth factor-1 mouse mutants. Hear Res. 2002;170(1-2):2-11.

42. Erickson SL, et al. ErbB3 is required for normal cerebellar and cardiac development: a comparison with ErbB2-and heregulin-deficient mice. Development. 1997;124(24):4999-5011.

43. Gassmann M, et al. Aberrant neural and cardiac development in mice lacking the ErbB4 neuregulin receptor. Nature. 1995;378(6555):390-394.

44. Riethmacher D, Sonnenberg-Riethmacher E, Brinkmann V, Yamaai T, Lewin GR, Birchmeier C. Severe neuropathies in mice with targeted mutations in the ErbB3 receptor. Nature. 1997;389(6652):725-730.

45. Stankovic K, et al. Survival of adult spiral ganglion neurons requires erbB receptor signaling in the inner ear. J Neurosci. 2004;24(40):8651-8661.

46. Kontaridis MI, Swanson KD, David FS, Barford D, Neel BG. PTPN11 (Shp2) mutations in LEOPARD syndrome have dominant negative, not activating, effects. J Biol Chem. 2006;281(10):6785-6792.

47. Martínez N, et al. Sprouty2 binds Grb2 at two different proline-rich regions, and the mechanism of ERK inhibition is independent of this interaction. Cell Signal. 2007;19(11):2277-2285.

48. Cazeneuve $\mathrm{C}$, et al. Identification of MEFVindependent modifying genetic factors for familial Mediterranean fever. Am J Hum Genet. 2000;67(5):1136-1143.

49. Knoblauch H, Schuster H, Luft FC, Reich J. A pathway model of lipid metabolism to predict the effect of genetic variability on lipid levels. J Mol Med.2000;78(9):507-515.

50. Slavotinek A, Biesecker LG. Genetic modifiers in human development and malformation syndromes, including chaperone proteins. Hum Mol Genet. 2003;12 Spec No 1:R45-R50.

51. Baxter R, Bannister LH, Dodson HC, Gathercole DV. Protein gene product 9.5 in the developing cochlea of the rat: cellular distribution and relation to the cochlear cytoskeleton. J Neurocytol. 1993;22(1):14-25.

52. Kim WY, et al. NeuroD-null mice are deaf due to a severe loss of the inner ear sensory neurons during development. Development. 2001;128(3):417-426.

53. Morris JK, et al. A disorganized innervation of the inner ear persists in the absence of ErbB2. Brain Res. 2006;1091(1):186-199.

54. Fagerheim T, et al. Identification of a new locus for autosomal dominant non-syndromic hearing impairment (DFNA7) in a large Norwegian family. Hum Mol Genet. 1996;5(8):1187-1191.

55. Takahashi A, et al. A novel potent tumour promoter aberrantly overexpressed in most human cancers. Sci Rep. 2011;1:15.

56. Riazuddin S, et al. Tricellulin is a tight-junction protein necessary for hearing. Am J Hum Genet 
2006;79(6):1040-1051.

57. DePristo MA, et al. A framework for variation discovery and genotyping using nextgeneration DNA sequencing data. Nat Genet. 2011;43(5):491-498.

58. Li H, Durbin R. Fast and accurate short read alignment with Burrows-Wheeler transform. Bioinformatics. 2009;25(14):1754-1760.

59. Thisse C, Thisse B. High-resolution in situ hybridization to whole-mount zebrafish embry- os. Nat Protoc. 2008;3(1):59-69.

60. Riddle RD, Johnson RL, Laufer E, Tabin C. Sonic hedgehog mediates the polarizing activity of the ZPA. Cell.1993;75(7):1401-1416.

61. Rodrigues GA, Falasca M, Zhang Z, Ong SH, Schlessinger J. A novel positive feedback loop mediated by the docking protein Gab1 and phosphatidylinositol 3-kinase in epidermal growth factor receptor signaling. Mol Cell Biol. 2000;20(4):1448-1459.
62. Adzhubei IA, et al. A method and server for predicting damaging missense mutations. Nat Methods. 2010;7(4):248-249.

63. Yue P, Melamud E, Moult J. SNPs3D: candidate gene and SNP selection for association studies. BMC Bioinformatics. 2006;7:166.

64. Schwarz JM, Rödelsperger C, Schuelke M, Seelow D. MutationTaster evaluates disease-causing potential of sequence alterations. Nat Methods. 2010;7(8):575-576. 